\title{
An atlas of mid-infrared dust emission in spiral galaxies *
} \author{
S. Madden ${ }^{1}$, and P. Mazzei ${ }^{3}$ \\ 1 DAPNIA/Service d'Astrophysique, CEA/Saclay, 91191 Gif-sur-Yvette Cedex, France \\ 2 Observatoire de Marseille, 2 place Le Verrier, 13248 Marseille Cedex 4, France \\ 3 Osservatorio Astronomico di Padova, 5 Vicolo dell'Osservatorio, 35122 Padova, Italy \\ 4 Joint Astronomy Center, 660 N. A'ohoku Place, Hilo, Hawaii 96720, USA \\ 5 Observatoire de Paris, 61 avenue de l'Observatoire, 75014 Paris, France
}

H. Roussel ${ }^{1}$, L. Vigroux ${ }^{1}$, A. Bosma ${ }^{2}$, M. Sauvage ${ }^{1}$, C. Bonoli ${ }^{3}$, P. Gallais ${ }^{1}$, T. Hawarden ${ }^{4}$, J. Lequeux ${ }^{5}$,

Received 3 November 2000 / Accepted 23 January 2001

\begin{abstract}
We present maps of dust emission at $7 \mu \mathrm{m}$ and $15 \mu \mathrm{m} / 7 \mu \mathrm{m}$ intensity ratios of selected regions in 43 spiral galaxies observed with ISOCAM. This atlas is a complement to studies based on these observations, dealing with star formation in centers of barred galaxies and in spiral disks. It is accompanied by a detailed description of data reduction and an inventory of generic morphological properties in groups defined according to bar strength and $\mathrm{HI}$ gas content.
\end{abstract}

Key words. atlas - galaxies: spiral - galaxies: ISM - infrared: ISM: continuum - infrared: ISM: lines and bands

\section{Introduction}

The mid-infrared maps presented here are part of a larger sample of nearby spiral galaxies analyzed in Roussel et al. (2001a and b: Papers I and II). Paper I primarily investigates the dynamical effects of bars on circumnuclear star formation activity, studied through dust emission at 7 and $15 \mu \mathrm{m}$, and proposes an interpretation of these midinfrared data in conjunction with information on molecular gas and stellar populations. Paper II deals with the use of mid-infrared fluxes as a star formation indicator in spiral disks. Observations belong to guaranteed time programs of ISOCAM, a camera operating between 5 and $18 \mu \mathrm{m}$ onboard the satellite ISO (described by Cesarsky et al. 1996). The sample consists of a first group of large and regular spirals, mainly barred, and of a second group of spiral galaxies belonging to the Virgo cluster. The angular resolution of $\approx 10^{\prime \prime}$ (HPBW), combined with spectroscopic information from the same instrument, enables a detailed view of the variations of two dust phases (unidentified infrared band carriers and very small grains, as characterized by e.g. Désert et al. 1990), tracing different physical conditions. A stellar contribution can also exist at $7 \mu \mathrm{m}$ (Boselli et al. 1998), but is negligible except in a few early-type galaxies.

Send offprint requests to: H. Roussel, e-mail: hroussel@cea.fr

* Based on observations with ISO, an ESA project with instruments funded by ESA Member States (especially the PI countries: France, Germany, The Netherlands and the UK) and with the participation of ISAS and NASA.
The general description of the sample and detailed photometric results are given in Paper I, where the spectra are also published. Here, we provide the details of observations and data reduction, total fluxes at 7 and $15 \mu \mathrm{m}$, a morphological description of galaxies grouped into appropriate categories and maps at $7 \mu \mathrm{m}$, along with optical images. The $15 \mu \mathrm{m}$ maps have very similar morphology and are therefore not shown. These images demonstrate the peculiar character of circumnuclear regions seen in the mid-infrared. Information on the $F_{15} / F_{7}$ colors of bright complexes and other selected regions is added.

All of the maps are published for the first time, except those of M51 (Sauvage et al. 1996) and NGC 6946 (Malhotra et al. 1996), which are both re-analyzed here.

\section{Observations}

All galaxies were observed with two broadband filters, LW3 centered at $15 \mu \mathrm{m}(12-18 \mu \mathrm{m})$ and LW2 centered at $7 \mu \mathrm{m}(5-8.5 \mu \mathrm{m})$, that we shall hereafter designate by their central wavelength. Maps covering the whole infraredemitting disk were built in raster mode, with sufficient overlap between adjacent pointings (half the detector field of view in most cases) to avoid border artifacts and to provide redundancy for spoiled exposures. In all cases, the field of view is large enough to obtain a reliable determination of the background level, except for NGC 4736 and 6744. NGC 5457, the largest spiral in the sample with an optical size of nearly $30^{\prime}$, was imaged with a combination of two overlapping observations made during different revolutions of the satellite. The pixel size is either $3^{\prime \prime}$ or $6^{\prime \prime}$, depending on the galaxy size, but for Virgo galaxies it 
was always $6^{\prime \prime}$, in order to increase the signal to noise ratio. The half-power/half-maximum diameters of the point spread function are respectively $6.8^{\prime \prime} / \simeq 3.1^{\prime \prime}$ at $7 \mu \mathrm{m}$ with a $3^{\prime \prime}$ pixel size, $9.5^{\prime \prime} / 5.7^{\prime \prime}$ at $7 \mu \mathrm{m}$ with a $6^{\prime \prime}$ pixel size, $9.6^{\prime \prime} / 3.5^{\prime \prime}$ at $15 \mu \mathrm{m}$ with a $3^{\prime \prime}$ pixel size and $14.2^{\prime \prime} / 6.1^{\prime \prime}$ at $15 \mu \mathrm{m}$ with a $6^{\prime \prime}$ pixel size. Table 1 summarizes the relevant information regarding the observations.

To estimate the relative importance of different emitting species in different galactic regions, spectral imaging between 5 and $16 \mu \mathrm{m}$ of the inner disks of five bright galaxies was also carried out. These observations are essential to interpret broadband imaging results. They consist of one pointing with two circular variable filters, from 16.5 to $9 \mu \mathrm{m}$ and from 9.6 to $5 \mu \mathrm{m}$, with a spectral resolution $\lambda / \Delta \lambda(F W H M)=35$ to 50 . The sampling varies between 0.24 and 0.45 of $\Delta \lambda(F W H M)$.

\section{Data analysis}

\subsection{Broadband filter maps}

Data reduction was performed using and adapting the ISOCAM Interactive Analysis package (CIA). The main difficulty and source of uncertainty is the slow time response of the detector, which is typical of cold photoconductors. Below $20 \mathrm{~K}$, the mobility of carriers becomes very slow, which generates long time constants, both in the bulk of the photoconductor and at contact electrodes. The typical time spent per pointing is $\approx 40-100$ s whereas in general several hundred seconds are needed to reach stabilization within $10 \%$ of the flux step.

Several causes of memory effects can be distinguished, although they always involve the same physics of the detector. We will use different designations for convenience: - (short-term) transients: they are due to the slow stabilization following a flux step (either upward or downward), after moving from one sky position to another; they are the origin of remnant images seen after observing a bright source;

- long-term transients: drifts and oscillations visible during the whole observation and influenced by previous history; contrary to short-term transients, no model exists to correct for them;

- glitch tails: slow return to the sky flux level following the deposition of energy in the detector by intense cosmic rays (this is an unpredictable effect);

- responsivity drops: they also follow cosmic ray impacts and produce "holes" which can last more than one hundred exposures (this is again an unpredictable effect).

The data reduction proceeded in the following way: 1 - The subtraction of the dark current follows a model which predicts its time evolution for even and odd rows of the detector. It accounts for variations along each satellite orbit and along the instrument lifetime ${ }^{1}$;

\footnotetext{
${ }^{1}$ It is described in "The ISOCAM dark current calibration report" by Biviano et al. (1998), which can be found at: http://www.iso.vilspa.esa.es/users/expl_lib/CAM_list. html
}

2 - If needed, we mask out the borders of the detector which are not sufficiently illuminated to allow a proper flat-fielding;

3 - The removal of cosmic ray impacts is complicated by the long-duration memory effects they can produce. The arrival of an energetic cosmic ray may be followed by either a slowly decreasing tail or a responsivity drop, as explained above. The vast majority of glitches are, however, of short duration (one to three exposures) and can be rejected by median filtering. To also remove small tails and plateaus induced by glitch pile-up, the exposures immediately surrounding exposures flagged by the deglitcher were examined and their flux level compared with that of adjacent non-flagged exposures, i.e. we iterated the median filtering once after rejecting the cosmic ray peaks. This filtering cannot blindly be applied everywhere. First, at a step between a faint and a bright illumination level and vice-versa, some real signal variation may be masked by the deglitcher. Therefore, the signal in the first and last exposures for each sky position was compared with that in the neighboring exposures for the same sky position, and flags cancelled if necessary. Second, during a pointing on a bright peaked source such as galactic circumnuclear regions, the noise includes, in addition to the readout and photon noises, high amplitude fluctuations which are approximately proportional to the signal and due to the satellite jitter. For the purpose of glitch detection, an additional noise component proportional to the signal was thus tuned to reproduce the jitter effects, and for very bright sources, defined by a flux threshold set above the background, fluctuations of $20 \%$ around the median flux were allowed. The memory effects following some glitches, for which no model exists, are temporarily set aside and examined at step 6 ;

4 - Short-term transients are corrected for by using the latest available technique taking into account the detector characteristics (Coulais \& Abergel 2000). The parameters of the model have been determined in low and uniform illumination cases only, but are likely to vary with signal intensity and are inexact in the presence of strong spatial gradients. Indeed, when switching from a moderate flux level to a bright resolved source, the correction hardly modifies the data. After a large amplitude flux step, the stabilization is, however, faster, and thus the last exposures are likely closer to the real flux than in the case of small flux steps. The readout histories of pixels imaging galactic nuclei or extranuclear bright complexes were carefully examined after step 7 to check whether the computed flux level was consistent with the level expected from the last valid exposures; if not, it was replaced with the mean signal in the last part of the readout history that was approximately flat as a function of time. Furthermore, after seeing a bright source, remnant images are not completely suppressed and can persist for several successive pointings; they are masked out at step 9;

5 - As long-term transients are not taken into account by the above correction, they were approximated and removed as one offset for each sky position, with respect to 
Table 1. Raster observations. Listed are the number of the figure showing the maps, the project name (B: Cambarre; S: Camspir; F: Sf $g l x$; V: Virgo), the pixel size, the number of pointings projected onto the raster map, the field of view, the number of exposures per pointing, the integration time per exposure, total on-target times at 15 and $7 \mu \mathrm{m}$, and total fluxes. When two figures are given, the first one corresponds to $15 \mu \mathrm{m}$ and the second one to $7 \mu \mathrm{m}$. For NGC 6946, observed with $6^{\prime \prime}$ pixels, each pointing is displaced from the previous by a fractional number of pixels, so that the pixel size of the projected map is $3^{\prime \prime}$

\begin{tabular}{|c|c|c|c|c|c|c|c|c|c|c|c|}
\hline name & Fig. & proj. & $\begin{array}{c}\text { pix. } \\
\left({ }^{\prime \prime}\right)\end{array}$ & $N_{\mathrm{p}}$ & $\begin{array}{c}\operatorname{map} \text { size } \\
\left({ }^{\prime \prime}\right)\end{array}$ & $n_{\exp }^{a}$ & $\begin{array}{r}t_{\text {int }} \\
(\mathrm{s})\end{array}$ & \multicolumn{2}{|c|}{ (s) } & \multicolumn{2}{|c|}{ (mJy) } \\
\hline N289 & $6 \mathrm{c}$ & B & 3 & 16 & 240 & 26 & 5.0 & 2086 . & 2086. & $327.8 \pm 25.6$ & $342.9 \pm 14.7$ \\
\hline N337 & $7 \mathrm{~b}$ & B & 3 & 16 & 240 & 26 & 5.0 & 2131. & 2137. & $297.9 \pm 24.0$ & $336.1 \pm 17.9$ \\
\hline N613 & $5 \mathrm{c}$ & $\mathrm{B}$ & 3 & 25 & 288 & 25 & 5.0 & 3170 . & 3170. & $1566.5 \pm 104.0$ & $1473.3 \pm 71.4$ \\
\hline N1022 & $7 \mathrm{a}$ & B & 3 & 16 & 240 & 26 & 5.0 & 2071. & 2076 . & $802.3 \pm 86.4$ & $444.4 \pm 45.3$ \\
\hline N1097 & $5 \mathrm{~d}$ & $\mathrm{~B}$ & 3 & 25 & 288 & 25 & 5.0 & 3119 . & 3114. & $2269.2 \pm 167.4$ & $2128.6 \pm 125.4$ \\
\hline N1365 & $5 \mathrm{~d}$ & $\mathrm{~S}$ & 6 & 16 & 480 & 61 & 2.1 & 1978. & 1984. & $4436.7 \pm 764.5$ & $3691.9 \pm 616.6$ \\
\hline N1433 & $5 \mathrm{~b}$ & B & 3 & 25 & 288 & 25 & 5.0 & 3165 . & 3175. & $355.3 \pm 41.0$ & $381.3 \pm 33.8$ \\
\hline N1530 & $5 \mathrm{~b}$ & B & 3 & 16 & 240 & 26 & 5.0 & 2137. & 2126. & $606.1 \pm 39.2$ & $573.9 \pm 39.1$ \\
\hline N1672 & $5 c$ & B & 3 & 25 & 288 & 25 & 5.0 & 3170 . & 3155. & $2020.5 \pm 123.0$ & $1985.0 \pm 129.2$ \\
\hline N4027 & $7 \mathrm{~b}$ & B & $\begin{array}{l}6 \\
3\end{array}$ & $\begin{array}{l}9 \\
4\end{array}$ & $\begin{array}{l}384 \\
132\end{array}$ & $\begin{array}{l}41,42 \\
42,41\end{array}$ & $\begin{array}{l}2.1 \\
2.1\end{array}$ & $\begin{array}{l}787 . \\
350 .\end{array}$ & $\begin{array}{l}787 . \\
359 .\end{array}$ & $676.7 \pm 95.5$ & $775.8 \pm 68.2$ \\
\hline N4535 & $6 \mathrm{~b}$ & B & $\begin{array}{l}6 \\
3\end{array}$ & $\begin{array}{c}16 \\
4\end{array}$ & $\begin{array}{l}480 \\
132\end{array}$ & $\begin{array}{c}27,20 \\
26\end{array}$ & $\begin{array}{l}5.0 \\
5.0\end{array}$ & $\begin{array}{c}2066 . \\
539 .\end{array}$ & $\begin{array}{c}2056 . \\
534 .\end{array}$ & $1127.9 \pm 181.4$ & $1136.6 \pm 68.9$ \\
\hline N4691 & $7 \mathrm{a}$ & B & $\begin{array}{l}6 \\
3\end{array}$ & $\begin{array}{l}4 \\
4\end{array}$ & $\begin{array}{l}288 \\
132\end{array}$ & $\begin{array}{l}41,42 \\
42,41\end{array}$ & $\begin{array}{l}2.1 \\
2.1\end{array}$ & $\begin{array}{l}359 . \\
352 .\end{array}$ & $\begin{array}{l}357 \\
354\end{array}$ & $795.9 \pm 185.6$ & $613.5 \pm 83.1$ \\
\hline N4736 (M94) & $6 \mathrm{~d}$ & $\mathrm{~S}$ & 6 & 9 & 288 & 20 & 5.0 & 917. & 922. & $4204.5 \pm 240.6$ & $3913.9 \pm 225.8$ \\
\hline N5194 (M51) & $6 \mathrm{~d}$ & $\mathrm{~S}$ & 3 & 100 & 663 & 27 & 2.1 & 5384. & 5390. & $8003.2 \pm 493.5$ & $8598.7 \pm 552.1$ \\
\hline N5236 (M83) & $6 \mathrm{a}$ & $\mathrm{S}$ & 6 & 49 & 768 & 21 & 5.0 & 4918. & 5155. & $20098.4 \pm 803.7$ & $18474.9 \pm 899.7$ \\
\hline N5383 & $5 \mathrm{a}$ & $\mathrm{B}$ & $\begin{array}{l}6 \\
3\end{array}$ & $\begin{array}{l}9 \\
4\end{array}$ & $\begin{array}{l}384 \\
156\end{array}$ & $\begin{array}{c}54 \\
54,52\end{array}$ & $\begin{array}{c}2.1 \\
2.1,5.0\end{array}$ & $\begin{array}{c}1024 . \\
459 .\end{array}$ & $\begin{array}{l}1022 . \\
1083 .\end{array}$ & $332.6 \pm 61.9$ & $350.2 \pm 62.1$ \\
\hline N5457 (M101) & & & & & & & & & & & \\
\hline $\begin{array}{l}\text { main } \dagger \\
\mathrm{E} \operatorname{arm} \dagger\end{array}$ & $6 \mathrm{c}$ & $\mathrm{S}$ & $\begin{array}{l}6 \\
6\end{array}$ & $\begin{array}{l}49 \\
25\end{array}$ & $\begin{array}{l}1302 \\
\times 972\end{array}$ & $\begin{array}{c}20,21 \\
25\end{array}$ & $\begin{array}{l}5.0 \\
5.0\end{array}$ & $\begin{array}{l}4949 . \\
3155 .\end{array}$ & $\begin{array}{l}4924 . \\
3140 .\end{array}$ & $5424.3 \pm 322.0$ & $6034.0 \pm 116.7$ \\
\hline $\mathrm{N} 6744$ & $6 \mathrm{~b}$ & $\mathrm{~S}$ & 6 & 16 & 480 & 25 & 5.0 & 2031. & 2041. & $1497.4 \pm 125.7$ & $2419.4 \pm 52.3$ \\
\hline N6946 & $6 \mathrm{a}$ & $\mathrm{F}$ & $6-3$ & 64 & 759 & 13,9 & $2.1,5.0$ & 1585. & 2872. & $10651.6 \pm 1767.2$ & $11648.8 \pm 678.6$ \\
\hline N7552 & $5 \mathrm{a}$ & $\mathrm{B}$ & 6 & 16 & 480 & 26 & 5.0 & 2091. & 2112. & & \\
\hline Virgo cluster & amplc & & 3 & 9 & 168 & 26 & 5.0 & 1184. & 1174. & $2767.6 \pm 193.7$ & $1826.2 \pm 168.5$ \\
\hline N4178 & $8 \mathrm{a}$ & $\mathrm{V}$ & 6 & 25 & 576 & 17,18 & 2.1 & 819. & 860. & $181.5 \pm 48.0$ & $228.5 \pm 24.6$ \\
\hline N4192 & $8 \mathrm{a}$ & $\mathrm{V}$ & 6 & 49 & 768 & 17,18 & 2.1 & 1528. & 1625. & $630.0 \pm 99.6$ & $900.8 \pm 68.3$ \\
\hline N4293 & $9 \mathrm{a}$ & $\mathrm{V}$ & 6 & 25 & 576 & 17,18 & 2.1 & 804. & 844. & $188.6 \pm 42.8$ & $159.5 \pm 25.3$ \\
\hline N4351 & $8 \mathrm{~b}$ & $\mathrm{~V}$ & 6 & 9 & 384 & 17,15 & 2.1 & 291. & 283. & $45.6 \pm 26.3$ & $52.6 \pm 8.7$ \\
\hline N4388 & $9 \mathrm{a}$ & $\mathrm{V}$ & 6 & 25 & 576 & 17,18 & 2.1 & 812. & 863. & $1008.2 \pm 244.0$ & $499.4 \pm 77.8$ \\
\hline N4394 & $9 \mathrm{~b}$ & $\mathrm{~V}$ & 6 & 16 & 480 & 16 & 2.1 & 480. & 478. & $139.0 \pm 41.0$ & $161.2 \pm 19.1$ \\
\hline N4413 & $9 b$ & $\mathrm{~V}$ & 6 & 9 & 384 & 17,16 & 2.1 & 302. & 281. & $93.0 \pm 31.4$ & $89.3 \pm 11.0$ \\
\hline N4430 & $8 \mathrm{~b}$ & $\mathrm{~V}$ & 6 & 9 & 384 & 17,16 & 2.1 & 294. & 279. & $98.0 \pm 23.5$ & $132.5 \pm 13.9$ \\
\hline N4438 & $9 \mathrm{c}$ & $\mathrm{V}$ & 6 & 49 & 768 & 17,18 & 2.1 & 1528. & 1629. & $209.1 \pm 34.7$ & $231.9 \pm 26.9$ \\
\hline N4450 & $9 \mathrm{c}$ & $\mathrm{V}$ & 6 & 25 & 576 & 17 & 2.1 & 791. & 844. & $169.7 \pm 42.5$ & $185.1 \pm 14.6$ \\
\hline N4491 & $9 \mathrm{~d}$ & $\mathrm{~V}$ & 6 & 9 & 384 & 17,15 & 2.1 & 310. & 287. & $81.1 \pm 25.2$ & $30.5 \pm 7.6$ \\
\hline N4498 & $9 \mathrm{~d}$ & $\mathrm{~V}$ & 6 & 16 & 480 & 16 & 2.1 & 493. & 478. & $94.6 \pm 19.2$ & $112.9 \pm 11.8$ \\
\hline N4506 & $9 \mathrm{e}$ & $\mathrm{V}$ & 6 & 9 & 384 & 17,16 & 2.1 & 294. & 277 . & $12.7 \pm 5.2$ & $21.1 \pm 9.8$ \\
\hline $\begin{array}{l}\text { N4567/ } \\
\text { N4568 }\end{array}$ & $8 \mathrm{c}$ & $\mathrm{V}$ & 6 & 25 & 576 & 17,18 & 2.1 & 779. & 829. & $\begin{array}{c}293.4 \pm 15.5 \\
1099.0 \pm 127.6\end{array}$ & $\begin{array}{c}317.9 \pm 16.4 \\
1074.7 \pm 64.8\end{array}$ \\
\hline N4569 & $9 \mathrm{e}$ & $\mathrm{V}$ & 6 & 64 & 864 & 16,18 & 2.1 & 1864. & 2108. & $939.3 \pm 125.1$ & $843.5 \pm 54.1$ \\
\hline N4579 & $9 f$ & $\mathrm{~V}$ & 6 & 36 & 672 & 17,19 & 2.1 & 1161. & 1306. & $619.2 \pm 85.1$ & $672.5 \pm 37.5$ \\
\hline N4580 & $9 f$ & $\mathrm{~V}$ & 6 & 9 & 384 & 17,16 & 2.1 & 298. & 279 & $103.9 \pm 24.2$ & $102.6 \pm 7.7$ \\
\hline $\begin{array}{l}\text { N4633/ } \\
\text { N4634 }\end{array}$ & $\begin{array}{l}8 \mathrm{c} / \\
8 \mathrm{~d}\end{array}$ & $\mathrm{~V}$ & 6 & 36 & 672 & 17,19 & 2.1 & 1123. & 1266. & $\begin{array}{c}30.0 \pm 9.5 \\
258.2 \pm 40.7\end{array}$ & $\begin{array}{c}30.3 \pm 9.1 \\
278.3 \pm 35.0\end{array}$ \\
\hline N4647 & $9 \mathrm{~g}$ & $\mathrm{~V}$ & 6 & 36 & 672 & 17,18 & 2.1 & 1127. & 1310. & $472.3 \pm 32.0$ & $474.3 \pm 17.2$ \\
\hline N4654 & $8 \mathrm{~d}$ & $\mathrm{~V}$ & 6 & 25 & 576 & 17,18 & 2.1 & 814. & 852. & $1018.6 \pm 78.4$ & $1049.4 \pm 42.9$ \\
\hline N4689 & $9 g$ & $\mathrm{~V}$ & 6 & 25 & 576 & 17,18 & 2.1 & 787. & 863. & $329.7 \pm 37.4$ & $340.9 \pm 16.3$ \\
\hline
\end{tabular}

$\bar{a}$ This is the minimum number of exposures. At the beginning of the observation for each filter, it is in general much larger, in order to get closer to stabilization.

$\dagger$ The map size given is that of the combination of the main map with the eastern arm map. 
the last sky position taken as a reference, step by step backwards and assuming that all pixels follow the same evolution. This is done by comparing the median of the highest possible number of pixels seeing the off-source sky in the current pointing and in any of the already-corrected pointings, and selecting the same set of pixels in both, since at this stage the images have not been flat-fielded. As it is treated as an offset, the correction does not modify the source flux but flattens the sky and allows better determination of the background level. It is similar to the long-term transient correction in the SLICE software of Miville-Deschênes et al. (2000);

6 - Short-term transients after a strong downward flux step which were imperfectly corrected at step 4, or glitch tails, left aside at step 3, and with the strongest time derivatives (hence the easiest to detect), were masked out by an automatic procedure. Drops below the background level, due to a responsivity drop after a glitch, were also masked out (without the need for strong time derivatives to design an automatic rejection);

7 - The valid exposures were averaged for each sky position;

8 - The flat-field response was systematically determined for each pixel as its mean value when illuminated by the background emission (masking the source), and then normalized by the mean in the $12 \times 12$ central pixels. When for a given pixel the number of useable values is too low (which happens if the pixel mostly images the source, or is often rejected due to glitches or memory effects), or when the error on the mean (computed from the dispersion) is higher than $5 \%$, the flat-field response of this pixel is not computed as the mean, but interpolated using the neighboring pixels and the default calibration flat-field, before applying the normalization. This method produces better results than the use of raw calibration files. These calibration data were employed only for NGC 4736 and NGC 6744, for which the field of view is too small to apply the above method (the background is hardly seen). For Virgo galaxies, which are small compared to the camera field of view and were mapped with numerous pointings, a time-variable flat-field was estimated, improving the final quality of the maps. The correction for long-term drifts (step 5) was then iterated once for all galaxies (this is again similar to the SLICE processing);

9 - The most conspicuous residual memory effects escaping rejection because they vary too slowly were removed manually in each sky position image. They were identified visually by comparing structure seen at the same sky coordinates in different overlapping pointings (corresponding to different sets of pixels with different histories), taking advantage of the spatial redundancy of the observations; 10 - The final map was built by projecting together all the individual images on a sky rectangular grid;

11 - The conversion from electronic units to spectral energy density units is made using the standard in-flight calibration data base.
Table 2. Parameters of the spectral observations. They include the pixel size, the ratio of the field of view to that of raster maps, the number of exposures per wavelength, the integration time per exposure and the total useful time

\begin{tabular}{|lccccc|}
\hline name & pix. size $\left({ }^{\prime \prime}\right)$ & frac. size & $n_{\text {exp }}$ & $t_{\text {int }}(\mathrm{s})$ & $T_{\text {tot }}(\mathrm{s})$ \\
\hline $\mathrm{N}_{13}{ }^{a}$ & 3 & 0.33 & 12 & 2.1 & 3960. \\
$\mathrm{~N}^{b} 097^{b}$ & 6 & 0.66 & $9-10$ & 2.1 & 3276. \\
$\mathrm{~N} 1365$ & 6 & 0.40 & 15 & 2.1 & 4918. \\
$\mathrm{~N} 5194$ & 6 & 0.29 & 15 & 2.1 & 4918. \\
$\mathrm{~N} 5236$ & 6 & 0.25 & 15 & 2.1 & 4918. \\
\hline
\end{tabular}

${ }^{a}$ At $3^{\prime \prime}$, no calibration flat-field in narrow filters was available, so we did not apply the division by $f f_{\text {filter }}$ (see last paragraph of Sect. 3.2).

${ }^{b}$ At the change of variable filter at $9.2 \mu \mathrm{m}$, a downward jump was visible in the whole field; to cancel it, we applied a unique offset to the second part of the spectrum.

\subsection{Spectral imaging}

In order to be able to decompose broadband observations according to the various emitting species, low-resolution spectra between 5 and $16 \mu \mathrm{m}$ were also obtained toward the inner $3^{\prime} \times 3^{\prime}$ or $1.5^{\prime} \times 1.5^{\prime}$ of five galaxies. Table 2 summarizes the parameters of these observations.

For spectral imaging, the principles of data reduction are the same as for broadband filters. Additional problems encountered in this type of observation are the following: - The observation is split into two slightly overlapping parts: from 16.5 to $9.2 \mu \mathrm{m}$ and then from 9.5 to $5 \mu \mathrm{m}$. The junction between the two circular variable filters, where the spectral transmission is low, can produce artefacts shortward of $9.2 \mu \mathrm{m}$ for a few wavelength steps, especially because of non-stabilization;

- There is a small displacement of the source from one wavelength to another, the total shift reaching up to two $3^{\prime \prime}$-pixels, which is due to the rotation and change of the circular variable filters. This effect was corrected for by fitting the shape of the galactic central regions by a Gaussian to measure their displacement on the array (for M 51, it was not possible and was done manually at the change between the two segments of the variable filter);

- Bright sources, such as galactic central regions, can create ghost images, due to reflections inside the instrument; in the case of NGC 1365, we could identify and isolate regions contaminated by obvious stray light ghosts. The flux collected by ghosts in a uniform illumination case is $\approx 20 \%$ (ISOCAM handbook $)^{2}$. For resolved sources, it depends on their position on the detector;

- The flat-field response used ignores any time or wavelength dependency. Nevertheless, this source of error is negligible compared with memory effects and the error on the determination of the background level;

2 ISO handbook volume III: CAM - the ISO camera, Siebenmorgen et al. 2000, SAI-99-057. It can be found at: http://www.iso.vilspa.esa.es/manuals/HANDBOOK/III/ cam_hb/ 


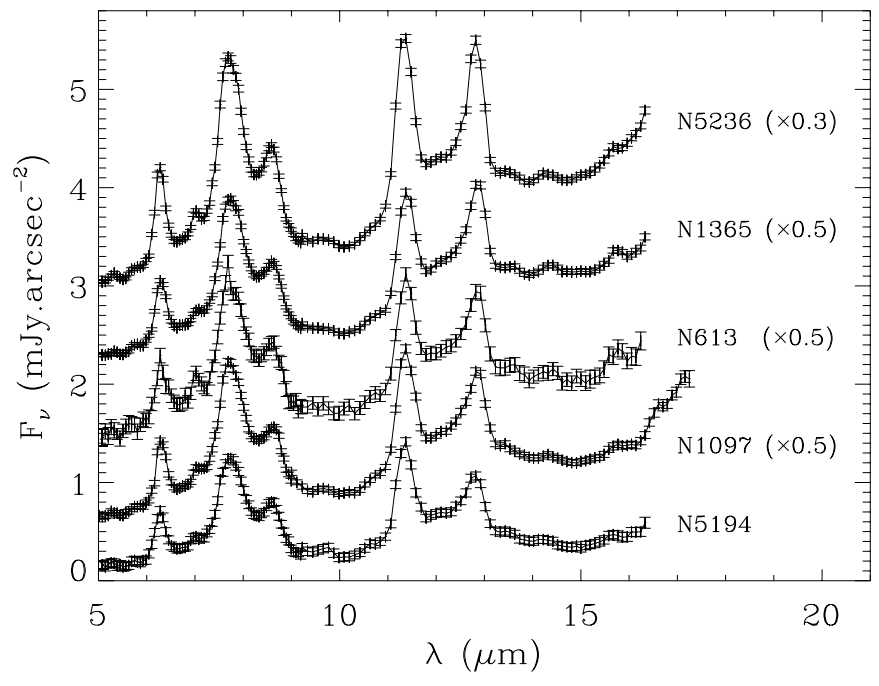

Fig. 1. Spectra of the central regions of the five galaxies observed in spectral imaging mode. They have been shifted for clarity by respectively $0.5,1.3,2.1$ and $2.9 \mathrm{mJy} \operatorname{arcsec}^{-2}$ and multiplied by the numbers indicated on the right. Error bars include the uncertainty on the zodiacal foregroung level, which is small with respect to the central source brightness

- Probably due to an inaccurate dark current subtraction amplified by the transient correction algorithm, the shortwavelength spectrum can be negative at faint signal levels; - As sources occupy the whole field of view and no spectra of empty regions were taken during the observations, the zodiacal foreground produced by interplanetary dust cannot be directly measured. We estimated it in the following way: we use a high signal to noise ratio calibration observation, from 16 to $5 \mu \mathrm{m}$ and then back from 5 to $16 \mu \mathrm{m}$ (which is useful to assess memory effects), of an empty part of the sky ${ }^{3}$. We apply an offset and a normalization to this zodiacal spectrum in order to obtain the maximum lower envelope to the average spectrum of the faintest pixels in our observation. The latter spectrum is composed of zodiacal light with superimposed faint emission bands (UIBs: see Paper I). Then, two bracketing zodiacal spectra with the same normalization are placed symmetrically; they are constrained to remain inside the dispersion limits of the observed spectrum, at least where it is not contaminated by UIBs. The minimum and maximum zodiacal spectra estimated in this way are subtracted from the galaxy spectrum, which produces an upper and a lower limit to the true spectrum. The minimum spectrum of the galaxy disk is also required to be positive, which tightens the bracketing of the zodiacal spectrum.

Due to the fact that the zodiacal light is several times higher than the disk spectrum (outside the bright central regions), the absolute intensity of the disk emission is subject to large uncertainties. However, since the zodiacal light spectrum is mainly featureless (it consists of a regularly rising part between 5 and $12 \mu \mathrm{m}$ and a slowly

\footnotetext{
3 The identification number of this observation, "TDT number" in the ISO archive, is: 06600401.
}

rising plateau from 11 to $16 \mu \mathrm{m}$ : see Fig. 1 in Paper I), the spectral shape of the disk emission is well determined; - Because of ghosts, the flat-field response was not handled as in broadband filter observations. The observed signal is the sum of the source, the background and their respective ghosts, multiplied by the flat-field response: $I=(S+b+g(S)+g(b)) \times f f$. However, the calibration flat-fields are also contaminated by ghosts: $f f_{\text {cal }}=$ $f f \times(1+g(b) / b)$. Therefore, we define the corrected signal as: $I_{\text {corr }}=\left(I-\right.$ zodi $\left.\times f f_{\text {cal }}\right) / f f_{\text {filter }}$, where zodi is the zodiacal spectrum estimated as described above and $f f_{\text {filter }}$ is a flat-field response measured with narrow filters, which does not contain any ghost. We then obtain $I_{\text {corr }}=S+g(S)$ : only ghosts due to the source remain.

The spectra resulting from the data processing described above are shown in Paper I (for central regions, disks, and the zodiacal emission), where they are discussed. In Fig. 1 we reproduce the spectra of the galactic central regions, with their noise and uncertainty on the zodiacal foreground (but no estimation of the errors due to memory effects was possible). Contribution from straylight ghosts can be of the order of 10-20\% (see Sect. 3.5).

\subsection{Photometry}

In some observations, the background is high compared to the source surface brightness, and it can be affected by inhomogeneities largely due to an incomplete correction for both short-term and long-term memory effects. The largest possible number of pixels has to be used, therefore, for a proper determination. In some other cases, a faint-level emission in spiral arms can be traced out to the map edge and likely continues outside the field of view. However, this emission is extremely faint and does not introduce any significant error in integrated fluxes. The only exceptions are NGC 4736 and NGC 6744, which are larger than the observation fields of view. For NGC 6744, $12 \%$ of the map in the southern part is blank, because the telescope did not move during 3 of the 16 programmed pointings. The given total fluxes are therefore lower limits. Other uncertain fluxes are those of the apparent pair NGC 4567/68, which has slightly overlapping disks in projection.

For all galaxies, the integrated flux was measured in concentric circles and traced as a function of the number of pixels, after excluding some sectors of the map where the galaxy extends to large distances, so as to see as much uncontaminated background as possible. The background was fitted in the linear part of this curve.

Total fluxes are given in Table 1 and other photometric results (see below) in Paper I.

\subsection{Separation of central regions from disks}

A common feature of dust emission distribution in our galaxies is a very strong central peak in a region of typically 1 to $3 \mathrm{kpc}$. Sub-structures are barely seen because the 
angular resolution is of the order of the size of these central regions, but can sometimes be suspected from variations in the infrared color $F_{15} / F_{7}$. We measured fluxes from this central condensation after defining its radius from the $7 \mu \mathrm{m}$ map. The galaxy center was first determined by fitting a two-dimensional Gaussian on an appropriate zone whenever possible. Otherwise, it was placed by visual criteria (for three galaxies). We computed the azimuthally averaged surface brightness profile in elliptical annuli to compensate for the inclination on the sky, using as far as possible orientation parameters derived from detailed kinematical analyses, otherwise from the position angle and axis ratio of outer isophotes. This profile was decomposed into a central region represented by a Gaussian and an inner disk represented by an exponential. The scale parameters were fitted on parts of the profile devoid of any obvious structure, such as rings or spiral arms, and each component was truncated at the radius where the exponential equals the Gaussian, which defined the photometric aperture radius for the circumnuclear region, $R_{\mathrm{CNR}}$.

Dilution effects were corrected for in two ways. The first consists of approximating central regions by point sources and dividing the fluxes measured inside $R_{\mathrm{CNR}}$ by the integral of the point spread function (PSF) inside the same aperture. The second one uses an iterative procedure. The image is first re-sampled on a fine grid (replacing each pixel by smaller pixels all taking the same value). The brightest pixel of the original sampling is looked for, the PSF centered on it is subtracted from both images with a small gain to ensure convergence of the process ( $5 \%$ here), and is then replaced in the fine-grid image with a Gaussian containing the same flux as the removed PSF, of $F W H M$ about half the pixel size. This procedure is analogous to the CLEAN algorithm used in radio astronomy (Högbom 1974). This is repeated until the residual image becomes reasonably uniform. For central regions not much more extended than the PSF, both estimates give equal results to a few percent; they differ in cases of a distribution flatter than the PSF or in the presence of sub-structure, such as a ring.

Fluxes attributable to the pure spiral disk were then defined as the difference between total fluxes and central region fluxes measured in this way.

\subsection{Errors and photometric consistency}

Photometric errors cannot be computed rigorously, due to the uncontrolled memory effects of the camera. The quoted uncertainties are therefore only indicative. They are the sum of three components:

- the readout and photon noises added quadratically, computed from the readout history of each pixel;

- the error on the background level fitted to the histogram of off-source pixels (which includes uncertainties due to residuals of dark current, flat-field, glitches, remnant images and long-term drifts), also added quadratically;
- the deviation from stabilization, grossly estimated from the dynamics of each pixel's response during the time spent on each pointing, as half the difference between the smoothed variation amplitude and the $99 \%$ confidence level readout and photon noise. This error component is added linearly over all the pixels imaging the galaxy, because memory effects cannot be assimilated to white noise.

The latter error is usually dominant, except for the faintest galaxies. We obtain typical errors of $\approx 10 \%$ at $7 \mu \mathrm{m}$ and $18 \%$ at $15 \mu \mathrm{m}$ (most galaxies having errors less than, respectively, 18 and 30\%). These do not take into account flux density calibration uncertainties, which are of the order of $5 \%$, with an additional $5 \%$ variation along each orbit (ISOCAM handbook).

The five galaxies observed in spectral imaging mode provide the opportunity to perform checks on the agreement between fluxes derived from independent observations. Since the wavelength coverage of our spectra ( 5 to about $16 \mu \mathrm{m}$ ) does not contain the whole $15 \mu \mathrm{m}$ band $(12-$ $18 \mu \mathrm{m})$, our comparison is limited to the $7 \mu \mathrm{m}$ band. We simulated observations in the LW2 band $(5-8.5 \mu \mathrm{m})$ using its quantum efficiency curve. The comparison with the photometry performed on the $7 \mu \mathrm{m}$ maps is shown in Fig. 2. The agreement is perfect for NGC 1365, but the spectra of the other four galaxies systematically overestimate $7 \mu \mathrm{m}$ fluxes with respect to raster maps. The amplitude of the deviation (23\% for NGC 613, $16 \%$ for NGC 1097, $12 \%$ for NGC 5194 and 17\% for NGC 5236) is of the order of what can be expected from stray light ghosts from the circumnuclear regions, which would be superimposed onto the source. This is consistent with the fact that an obvious ghost can be identified (outside the central regions) only in the case of NGC 1365, whose circumnuclear region is thus likely free from the ghost it generates. It should then be remembered that photometry performed on raster maps is more reliable. See also Förster-Scheiber et al. (2001) for a photometric comparison between ISOCAM and ISOSWS spectra.

Second, we can compare our results with independent measurements from the same ISOCAM raster observations (differing in the applied data reduction and photometry), for some galaxies of the sample analyzed by other individuals. In the Cambarre and Camspir samples, there is only one such galaxy, M 83, bright and very extended, for which Vogler et al. (2001) give $F_{15}=20.2 \mathrm{Jy}$ and $F_{7}=19.3 \mathrm{Jy}$, that is to say respectively 1.005 and 1.04 times the values obtained by us: the difference is well within the error bars. The fluxes of Virgo galaxies from Boselli et al. (1998) have not yet been published, so that we cannot check their consistency with ours.

We have also processed the 7 and $15 \mu \mathrm{m}$ maps of several galaxies from the $S f_{-} g l x$ observation program (PI G. Helou), selected from the ISOCAM public archive and added to our sample for the analysis presented in Paper I. The maps are not presented here since they have been published in Dale et al. (2000) (DSH). The results of our 


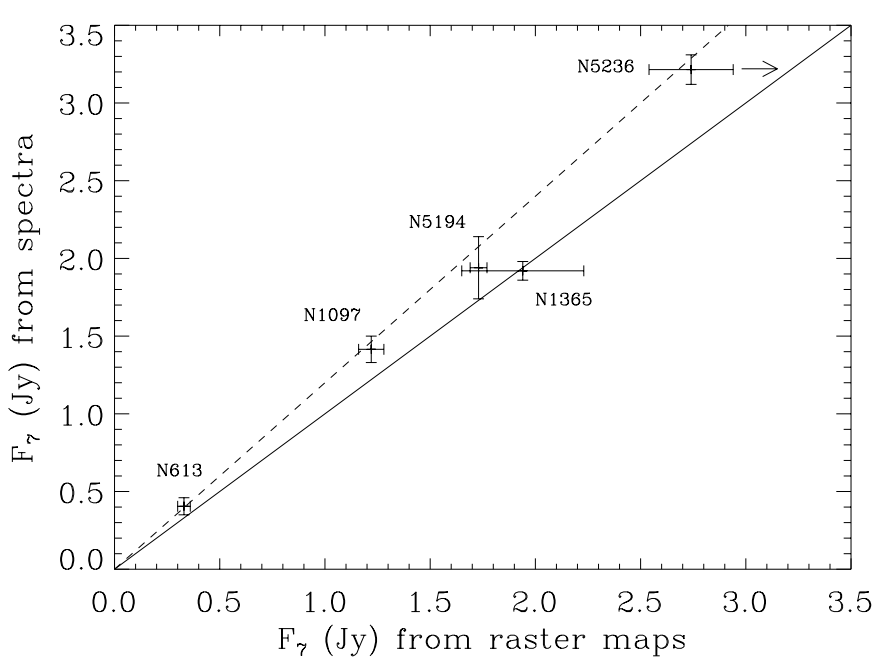

Fig. 2. $7 \mu \mathrm{m}$ fluxes simulated from the spectra (ordinate) versus $7 \mu \mathrm{m}$ fluxes measured in maps (abscissa). The apertures used are centered on the galactic nuclei and encompass the entire circumnuclear regions (chosen diameters of respectively $24^{\prime \prime}, 48^{\prime \prime}, 48^{\prime \prime}, 84^{\prime \prime}$ and $48^{\prime \prime}$ for NGC 613, 1097, 1365, 5194 and $5236)$. Note that the nucleus of NGC 5236 is slightly saturated in the $7 \mu \mathrm{m}$ map. Error bars include deviation from stabilization for raster maps and the uncertainty on the zodiacal foreground level for spectra. The solid line indicates $y=x$ and the dashed line $y=1.2 x$

own photometry are given in Paper I. Figure 3 shows the confrontation of our fluxes with those of DSH. NGC 6946, which is brighter by one order of magnitude than the other galaxies, is not included, for clarity (DSH obtain fluxes within respectively $8 \%$ and $4 \%$ of ours at 15 and $7 \mu \mathrm{m}$ for this object). As seen in Fig. 3, whereas the agreement at $15 \mu \mathrm{m}$ is reasonable (for all galaxies except three, the difference is less than $15 \%$ ), the results at $7 \mu \mathrm{m}$ are dramatically discrepant, our fluxes being systematically higher than those of DSH. As a result, $F_{15} / F_{7}$ colors computed by DSH are always higher than ours. To investigate the origin of this discrepancy, we performed some tests on NGC 986, an extended galaxy with bright circumnuclear regions, for which $F_{15}(\mathrm{DSH})=1.02 F_{15}$ (us) and $F_{7}(\mathrm{DSH})=0.64 F_{7}$ (us).

We substituted each step of our data reduction successively, leaving all the other steps unchanged, according to what we thought DSH had done from the information they give on their data processing: use of calibration files for the dark current; use of the deglitcher described by Starck et al. (1999) with default parameters and without any subsequent masking; use of the "fit3" routine with default parameters for short-term transient correction; suppression of the long-term transient correction; and use of calibration files for the flat-field.

We would advise against the empirical "fit3" routine for photometric purposes (it always finds a solution and artificially produces corrected readout histories which are flat, but this does not ensure that it converges toward the right value). Thus we initially thought that photometric discrepancies would be attributable to the short-term
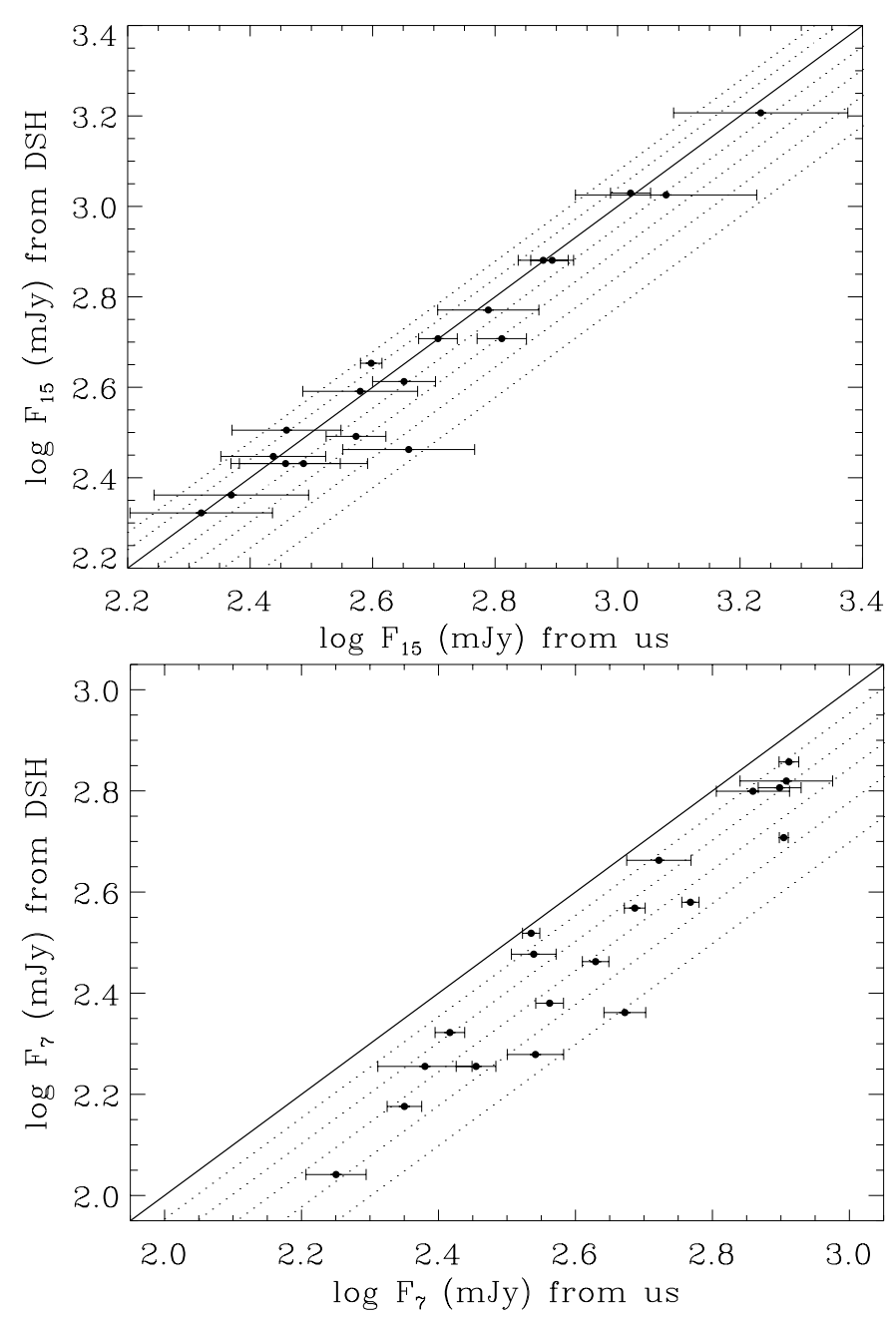

Fig. 3. Comparison of our photometric results at $15 \mu \mathrm{m}$ (top) and $7 \mu \mathrm{m}$ (bottom) with those of Dale et al. (2000) (DSH). Our error bars include the three components described above but not the systematic flux density calibration uncertainty, which acts equally on both sets of data. The solid line indicates $y=x$ and the dotted lines $y=(1+\alpha) x$, with $\alpha$ varying by steps of 0.1

transient correction. However, none of the changes listed above is sufficient to account for the large difference in the $7 \mu \mathrm{m}$ fluxes. We also tried to reproduce the photometric method of DSH, but this also can be excluded as the major source of the discrepancy.

As a last possibility, DSH mention that besides using calibration data, they also built flat-fields using the observations themselves, by computing the median of the images - but apparently without previously masking the areas where the galaxy falls -; we do not know to which galaxies exactly this applies and in which instances they have instead used calibration files. We have thus explored this track and created such a flat-field for NGC 986. We find that this is the only way we can recover the $7 \mu \mathrm{m}$ flux given by $\mathrm{DSH}$.

However, we emphasize that flat-fields should not be built from the observations without masking the source, especially for an extended galaxy with bright central regions such as NGC 986, on a small raster: when doing 
so, one strongly overestimates the flat-field response at all the places where bright sources are observed (for the $2 \times 2$ raster of NGC 986 for instance, the nucleus is seen at four places on the detector, and still at three places if the first sky position is removed, as DSH have done). As a consequence, after dividing by such a "flat-field", the final flux of the galaxy is severely underestimated. If indeed DSH have proceeded this way, this could explain why their fluxes are systematically lower than ours. If the same processing holds for $15 \mu \mathrm{m}$ maps, we speculate that the discrepancy with our $15 \mu \mathrm{m}$ fluxes is reduced due to a compensation effect by the background: at $15 \mu \mathrm{m}$, the zodiacal emission is much higher than at $7 \mu \mathrm{m}$ with respect to the galaxy flux (by a factor 5-6). Since the contrast between the background and the galaxy is much lower, the quality of the flat-field response is less affected than at $7 \mu \mathrm{m}$.

As a further check, one of us (M. Sauvage) independently reduced the maps and measured the fluxes of NGC 986 by a method different to that explained in Sect. 3.3. At $7 \mu \mathrm{m}$, allowing the isophotal detection limit to vary between 0 and 3 times the background dispersion above the background level, the resulting flux is within $-6 \%$ and $+8 \%$ of our tabulated flux, and the preferred measurement in view of the extension of the galaxy is at $-4 \%$. At $15 \mu \mathrm{m}$, these numbers are respectively $-14 \%$ and $+13 \%$, and $-7 \%$. Hence, our various estimates are in good agreement with each other.

\section{Maps}

For each galaxy are shown, from top to bottom: an optical map from the Digitized Sky Survey (with a better contrast inset from the second generation survey, if available and if the large-scale image is saturated); the $7 \mu \mathrm{m}$ map, to which we applied a transfer function $I^{n}$, where $0.4 \leq n \leq 1$, so as to make both faint and bright structures visible with the grey scale; $F_{15} / F_{7}$ colors of a few regions, selected for being regular and bright at 7 or $15 \mu \mathrm{m}$, which are shown as an illustration of the constancy of colors in disks, except in a few resolved star formation complexes and in circumnuclear regions. For these color measurements, $7 \mu \mathrm{m}$ maps were first convolved to the $15 \mu \mathrm{m}$ angular resolution. As the astrometry is not more precise than $\approx 10^{\prime \prime}$ (the absolute accuracy of ISO, $1-2^{\prime \prime}$, is degraded by ISOCAM's lens wheel jitter), the displacement between both maps was measured on central regions, by fitting them with a Gaussian when possible. For some Virgo galaxies not observed at 7 and $15 \mu \mathrm{m}$ in concatenated mode but during different revolutions, there is a rotation between the two maps of up to $5^{\circ}$, which was estimated by fitting the few usable regular complexes with Gaussians. Apertures were fixed by the angular resolution: we chose $12^{\prime \prime}$ for observations with a $3^{\prime \prime}$ pixel size, and $18^{\prime \prime}$ for a $6^{\prime \prime}$ pixel size, i.e. $\approx 1.25$ times the half-power diameter of the point spread function. We first give the colors of the circumnuclear region inside the radius defined as explained in Sect. 3.4 (marked "C"), and of the whole averaged disk (marked
"D"). Regions in the bar are noted "B" and regions in arms, arcs or rings are noted "A". In some cases, the color of the central region inside the resolution unit is also given ("Cr"). Fluxes are expressed in mJy, so that to obtain a true ratio of powers emitted inside the $12-18 \mu \mathrm{m}$ and 5-8.5 $\mu \mathrm{m}$ bandpasses (and not a flux density ratio), one should multiply $F_{15} / F_{7}$ by $\approx 0.42$.

Errors on average disk colors include only photon and readout noises and the uncertainty on the background level; for all other color measurements, they additionally contain an estimate of the errors due to incomplete stabilization. We again warn the reader that the latter error component cannot be derived rigorously: what follows is an attempt to provide an order-of-magnitude estimate. Since transients are of a systematic nature (the response of the camera to a given input is perfectly reproducible) and tend to behave coherently in a well defined region (a brightness peak or minimum), we treated them in the following way:

- We call $m_{i}^{\lambda}$ the estimated uncertainty due to memory effects in the pixel $i$ of the final map at the wavelength $\lambda$, derived as explained in the begining of Sect. 3.5. $R^{\lambda}$ is the ensemble of pixels belonging to the region of interest. Then the stabilization error on the true total flux $F^{\lambda}$ of that region is

$M^{\lambda}=\sum_{i \in R^{\lambda}} m_{i}^{\lambda}$

- We consider two cases: either both resulting errors at 7 and $15 \mu \mathrm{m}$ are treated positively, i.e. fluxes are overestimated (we measure $F_{\text {mes }}^{\lambda} \approx F^{\lambda}+M^{\lambda}$ instead of $F^{\lambda}$ ), or both are treated negatively, i.e. fluxes are underestimated $\left(F_{\text {mes }}^{\lambda} \approx F^{\lambda}-M^{\lambda}\right)$. We assume the same trend in both bands because memory effects are systematic in nature, and we consider it equally probable that fluxes are overestimated or underestimated in the case of an upward flux step as well as in the case of a downward step, because the detector response can oscillate as a function of time; - An asymmetric error bar on the color is then directly derived. Let us call $\mathcal{M}_{+}^{\mathrm{c}}$ and $\mathcal{M}_{-}^{\mathrm{c}}$ the bounds of this error bar: the measured color is noted $\left(F_{\text {mes }}^{15} / F_{\text {mes }}^{7}\right)_{-\mathcal{M}_{-}^{c}}^{+\mathcal{M}_{+}^{c}}$. If true fluxes are overestimated,

$$
\begin{aligned}
\frac{F_{\mathrm{mes}}^{15}}{F_{\mathrm{mes}}^{7}} & =\frac{F^{15}}{F^{7}} \times \frac{1+M^{15} / F^{15}}{1+M^{7} / F^{7}} \\
& \approx \frac{F^{15}}{F^{7}} \times\left(1+M^{15} / F^{15}-M^{7} / F^{7}\right)
\end{aligned}
$$

using a limited development. If $D_{\mathrm{p}}^{\mathrm{c}}$ is the deviation from the true color $\left(F_{\text {mes }}^{15} / F_{\text {mes }}^{7}-F^{15} / F^{7}\right)$ induced by an overestimation of fluxes and $D_{\mathrm{n}}^{\mathrm{c}}$ the deviation resulting from an underestimation of fluxes, one obtains

$$
D_{\mathrm{p}}^{\mathrm{c}}=\frac{F_{\mathrm{mes}}^{15}-M^{15}}{F_{\mathrm{mes}}^{7}-M^{7}} \times\left[\frac{M^{15}}{F_{\mathrm{mes}}^{15}-M^{15}}-\frac{M^{7}}{F_{\mathrm{mes}}^{7}-M^{7}}\right]
$$

and similarly

$$
D_{\mathrm{n}}^{\mathrm{c}}=\frac{F_{\mathrm{mes}}^{15}+M^{15}}{F_{\mathrm{mes}}^{7}+M^{7}} \times\left[-\frac{M^{15}}{F_{\mathrm{mes}}^{15}+M^{15}}+\frac{M^{7}}{F_{\mathrm{mes}}^{7}+M^{7}}\right] .
$$


- There are four possibilities:

If $D_{\mathrm{p}}^{\mathrm{c}}>0$ and $D_{\mathrm{n}}^{\mathrm{c}}<0$ then $\mathcal{M}_{-}^{\mathrm{c}}=D_{\mathrm{p}}^{\mathrm{c}}$ and $\mathcal{M}_{+}^{\mathrm{c}}=-D_{\mathrm{n}}^{\mathrm{c}}$. If $D_{\mathrm{p}}^{\mathrm{c}}<0$ and $D_{\mathrm{n}}^{\mathrm{c}}>0$ then $\mathcal{M}_{+}^{\mathrm{c}}=-D_{\mathrm{p}}^{\mathrm{c}}$ and $\mathcal{M}_{-}^{\mathrm{c}}=D_{\mathrm{n}}^{\mathrm{c}}$. If $D_{\mathrm{p}}^{\mathrm{c}}>0$ and $D_{\mathrm{n}}^{\mathrm{c}}>0$ then $\mathcal{M}_{-}^{\mathrm{c}}=\max \left[D_{\mathrm{p}}^{\mathrm{c}}, D_{\mathrm{n}}^{\mathrm{c}}\right]$ and $\mathcal{M}_{+}^{\mathrm{c}}=0$.

If $D_{\mathrm{p}}^{\mathrm{c}}<0$ and $D_{\mathrm{n}}^{\mathrm{c}}<0$ then $\mathcal{M}_{+}^{\mathrm{c}}=-\min \left[D_{\mathrm{p}}^{\mathrm{c}}, D_{\mathrm{n}}^{\mathrm{c}}\right]$ and $\mathcal{M}_{-}^{\mathrm{c}}=0$.

Maps are organized as follows:

Figure 5: strongly barred galaxies arranged in order of decreasing $D_{\text {bar }} / D_{25}$, the ratio of the deprojected bar diameter to the disk diameter;

Figure 6: galaxies with weak or no bar arranged in the same order;

Figure \%: the peculiar galaxies;

Figure 8: all galaxies of the Virgo cluster sample whatever the bar strength which are not HI-deficient;

Figure 9: HI-deficient Virgo galaxies.

Maps are oriented with north to the top and east to the left.

\section{Morphological properties}

In the following, we grouped galaxies according to their most striking property. The well-resolved regular spirals of the first subsample were arranged in order of decreasing $D_{\text {bar }} / D_{25}$, the ratio of the deprojected bar diameter to the disk major diameter at the blue isophote $25 \mathrm{mag} \operatorname{arcsec}^{-2}$. Virgo galaxies are resolved with less detail, are more frequently highly inclined and intrinsically smaller and fainter, not allowing a meaningful measure of $D_{\mathrm{bar}} / D_{25}$. They are separated into two groups differentiated by interactions with the cluster environment: galaxies with a normal Hi content and Hi-deficient galaxies.

Beyond the complexity and distinctive features of all galaxies shown here, we choose to emphasize in this section the generic properties found within each group and do not describe in depth each individual object. The morphological types from the RC3 (de Vaucouleurs et al. 1991) are indicated.

We determined the size of the infrared emitting disk (at $7 \mu \mathrm{m}$ because the sensitivity is better than at $15 \mu \mathrm{m}$ ), defined as the diameter of the isophote $5 \mu \mathrm{Jy} \operatorname{arcsec}^{-2}$. This is the deepest level that can be reasonably reached for all galaxies of the sample, corresponding to between one and five times the background $1 \sigma$ uncertainty (except for NGC 5194 which has a higher background noise). This level is very similar to the sensitivity reached by Rice et al. (1988) in $12 \mu \mathrm{m}$ IRAS maps. The measure was performed on azimutally averaged surface brightness profiles, taking into account the orientation and inclination of the galaxy. We cannot see any difference in the mid-infrared size to optical size ratio between barred and non-barred galaxies, but this ratio tends to increase from early-type to latetype galaxies. More fundamental systematics exist as a function of gas content, which will be described in Sect. 6 . Indeed, the likely reason for the observed dependence on Hubble type is the existence of a classification bias for
HI-deficient galaxies: they are mostly classified as early type, due to the abnormal aspect of their spiral arms, and since the bulge-to-disk ratio criterion plays a minor role in the classification compared to the resolution of spiral arms into star formation complexes.

\subsection{Well resolved galaxies}

\section{- Strongly barred spirals:}

NGC 5383 (SBb), 7552 (SBab), 1433 (SBab), 1530 (SBb), 613 (SBbc), 1672 (SBb), 1097 (SBb) and 1365 (SBb).

The circumnuclear region is extremely bright in the mid-infrared - always brighter than any complex in the bar or the arms. This is particularly obvious in NGC 7552, whose bar is relatively faint and where only a hint of the eastern arm is present, whereas emission from the large central region is very intense. All galaxies of this category are known to possess a nuclear spiral or ring, and sometimes additionally a nuclear bar. All except NGC 1433 have a hot-spot nucleus, i.e. giant star formation complexes within the central structure. We give here a brief summary of nuclear morphologies:

- NGC 5383: nuclear spiral (Buta \& Crocker 1993) with hot spots (Sersic 1973);

- NGC 7552: nuclear ring (Feinstein et al. 1990) with hot spots - although the nucleus is classified amorphous by Sersic \& Pastoriza (1965), bright complexes are clearly visible in the radio continuum maps of Forbes et al. (1994) -; Dottori \& Pastoriza (1986) infer from a population synthesis of stellar absorption lines a cycle of three or four successive starbursts;

- NGC 1433: nuclear ring and nuclear bar (Buta 1986), amorphous nucleus (Sersic \& Pastoriza 1965);

- NGC 1530: nuclear spiral (Buta \& Crocker 1993) with hot spots (Sersic 1973);

- NGC 613: nuclear spiral and nuclear bar (Jungwiert et al. 1997), hot spots (Sersic 1973);

- NGC 1672: nuclear ring with hot spots (StorchiBergmann et al. 1996), although Sersic \& Pastoriza (1965) include it among amorphous nuclei;

- NGC 1097: circumnuclear starburst ring of diameter $20^{\prime \prime} \approx 1.5 \mathrm{kpc}$ with hot spots, nuclear bar (Friedli et al. 1996). The ring is well resolved in the mid-infrared;

- NGC 1365: nuclear spiral and nuclear bar (Jungwiert et al. 1997), hot spots (Morgan 1958).

The bar is detected in all galaxies but NGC 1433, which, besides its central region, displays only very faint and patchy emission; in NGC 1365, it is detected but extremely diffuse.

We also note that the mid-infrared emission of the bar generally consists of thin bands (as opposed to the stellar bar which is more oval), shifted towards the leading edge (if one assumes that spiral arms are trailing), in agreement with the response of other gas tracers to the bar potential. However, these bands do not coincide strictly with dust lanes seen in optical images: dust which is conspicuous in optical starlight absorption is not the same as 
dust seen in mid-infrared emission. In fact, absorbing dust lanes are signatures of gas density enhancements, at the location of shocks (Athanassoula 1992). As these shocks are strongest in the inner parts of the bar, near the central region, absorption dust lanes are also better developed and show higher contrast in the inner bar, independently of the brightness of the underlying stellar continuum (this is particularly well seen in NGC 1530 and 1672); conversely, the infrared emission is systematically brightest in the outer parts of the bar and diffuse near the circumnuclear region. The same argument excludes significant collisional excitation of dust in shocks: grains are heated by photons, including in regions of large-scale shocks.

A further sign of the different nature of absorption and emission dust lanes is the aspect of the bar of NGC 1365: whereas absorption dust lanes show high contrast and are displaced far from the bar major axis toward the leading edge, the infrared emission is extremely diffuse and rather symmetric about the major axis.

Star formation complexes are generally seen in the bar at varying places, between mid-distance from the nucleus to the end of the bar (see for instance the bright knots in NGC 7552 and 1530). Such complexes inside the bar are also commonly found in $\mathrm{H} \alpha$ observations (García-Barreto et al. 1996; Martin \& Friedli 1997). Emission is always enhanced at the junction between the bar and spiral arms, even in NGC 1433. This has been interpreted as an effect of orbit crowding, since it is the place where elongated orbits subtended by the bar meet near-circular orbits of the disk (Kenney \& Lord 1991). This region therefore favours star formation, unlike the inner bar where the high-velocity shocks are likely to prevent it (Tubbs 1982; Athanassoula 1992; Reynaud \& Downes 1998).

In some galaxies (NGC 1530, 1365 and to a lesser extent NGC 5383), spiral arms are clearly seen to begin in advance of the bar, i.e. to continue beyond the junction with the bar towards the leading edge, which is never the case for weakly barred galaxies. This could be due to a decoupling between the bar and spiral waves, i.e. different pattern speeds. Spiral arms generally make sharp angles with the bar (except the eastern arms of NGC 7552, 613 and 1672), which is also characteristic of strong bars, as already noted by Prendergast (1983).

The inner parts of arms show in most cases much star formation, as evidenced by bright emission and knots both in the optical and in the mid-infrared (exceptions are NGC 7552, whose western arm is not detected at all and whose eastern arm is hardly seen, and NGC 1433, whose entire inner ring is seen near the detection limit). Nevertheless, there seems to be a maximum radius encompassing sites of reasonable star formation rates, outside of which spiral arms abruptly become fainter, both in optical and infrared images. The clearest and most symmetric example is NGC 1365, but this remark is valid for all. The exhaustion of outer arms could be a signature of the corotation between the spiral wave and the gas, but it can also be related to the fact that all the strongly barred galaxies presented here are of early type (between SBab and SBb, with one SBbc, NGC 613). This is not a bias in the sample: long bars are found predominantly among early types (Athanassoula \& Martinet 1980; Martin 1995).

We finally note the presence of an inner plateau or lens of diffuse infrared emission delineating a sort of inner pseudo-ring in all galaxies of this group, again except NGC 7552 and 1433. In NGC 1530, the plateau is also seen in diffuse $\mathrm{H} \alpha$ emission and the pseudo-ring materialized by $\mathrm{H} \alpha$ knots (Regan et al. 1996). In NGC 613, 1672 and 1097, a real lens is seen in the optical and further outlined by secondary tightly wound arms or incomplete arcs, best seen in the infrared.

\section{- Weakly or non barred spirals:}

NGC 5236 (SABc), 6946 (SABcd), 4535 (SABc), 6744 (SABbc), 289 (SBbc), 5457 (SABcd), 4736 (SAab), 5194 (SAbc).

In weakly barred spirals, the central region is bright in the mid-infrared, but contrary to strong bar centers, it is relatively small and can be rivaled in brightness by complexes in the arms. The centers of NGC 5236 and 6946 are particularly intense. Several of these galaxies also contain nuclear rings, but none seems to harbor hot spots comparable to those seen in galaxies of the first group. Here is a list of nuclear morphologies:

- NGC 5236: nuclear ring (Buta \& Crocker 1993), amorphous nucleus (Sersic \& Pastoriza 1965);

- NGC 6946: nuclear bar (Zaritsky \& Lo 1986);

- NGC 4535: nuclear ring (Buta \& Crocker 1993);

- NGC 6744, 289 and 5457: no remarkable structure;

- NGC 4736: weak nuclear bar (Möllenhoff et al. 1995);

- NGC 5194: nuclear ring (Buta \& Crocker 1993), which is seen in the mid-infrared.

The bar is detected in all SABs except NGC 6744, which is completely devoid of infrared emission between the nucleus and the inner ring, much like NGC 1433. Unlike strong bars, weak bars do not contain infrared knots: their emission is rather unstructured. They consist of thin lanes displaced towards the leading edge, just as in strong bars, but are more curved and smoothly connected to spiral arms, in agreement with the notations of Prendergast (1983).

In NGC 6946, the bar is rather a fat oval distribution of stars, inside which a spiral arm can be traced at the north. The bar that we see in the infrared is shorter and rotated by as much as $\approx 35^{\circ}$ to the leading side of the stellar oval. It does not coincide with the small northern arm but corresponds well to the $\approx 1^{\prime}$ molecular bar-like distribution (Ball et al. 1985; Regan \& Vogel 1995), confirmed kinematically by Bonnarel et al. (1988), and in which is embedded a nuclear bar rotated by a further angle of $\approx 15^{\circ}$ to the leading side.

Zones at the junction between the bar and spiral arms are much less conspicuous than in strongly barred galaxies (except the SE junction in NGC 289 which is bright). Brightness peaks are found further out along the arms (see in particular NGC 4535). Dust emission follows 
remarkably closely the spiral structure at very large distances from the center. Note for instance the strong similarity between optical and infrared images of NGC 4535, where even the slight brightness enhancement at the northern edge of the disk, likely due to compression by the intracluster gas, is detected at 7 and $15 \mu \mathrm{m}$. There is no sharp brightness decrease beyond some radius as in strongly barred spirals, except in NGC 289. This galaxy appears to be the closest of its category to strongly barred galaxies (bright knot at the end of the bar, rather sharp angle of spiral arms with the bar, slight advance of the arms on the bar, abrupt decrease in surface brightness between an inner disk and an outer disk), despite its rather short bar. However, we note that the outer disk, whose size defines the normalization to the bar size, is extremely tenuous, unlike in the other spirals of this group.

NGC 4736, although classified SA, has an oval distorsion revealed by its velocity field (Bosma et al. 1977), hence a weak bar. Its metallicity radial gradient is typical of SAB galaxies and could perhaps result from the past mixing effects of a stronger bar now dissolved (Martin \& Belley 1997).

\section{- Peculiar spirals:}

NGC 1022 (SBa) and 4691 (SB0/a).

NGC 4691 is described as an "irregular galaxy of the M 82 type" by Sandage (1961) and then as amorphous (Sandage \& Bedke 1994), based on the chaotic, "intricate and extensive dust pattern in the central regions" which is similar to that in some starburst galaxies such as M 82 and suggests "galactic fountain activity". This interpretation is supported by the spectroscopic data of García-Barreto et al. (1995), whose I-band and $\mathrm{H} \alpha$ images also reveal a peculiar central structure with four knots. The two strongest $\mathrm{H} \alpha$ knots are resolved in our mid-infrared images, and are surrounded by two concentrations of molecular gas aligned with them, but at nearly twice the distance from the center (Wiklind et al. 1993). In the mid-infrared, we detect only the central structures and not the bar or the rest of the disk.

NGC 1022 is also peculiar and resembles NGC 4691 in many aspects. Both have extremely faint and smooth arms and a chaotic system of dust filaments, in all the interior of the inner ring for NGC 1022 (Sandage \& Bedke 1994). NGC 1022 has a very short bar for a SBa galaxy. All interstellar tracers are highly concentrated in a small circumnuclear region (García-Barreto et al. 1991; Hameed \& Devereux 1999) and we detect only this central region of size $<10^{\prime \prime} \approx 1 \mathrm{kpc}$ in the mid-infrared. The morphology of both galaxies is suggestive of a past merger.

\section{- Magellanic barred spirals:}

NGC 337 (SBd) and 4027 (SBdm).

These are the two latest-type galaxies of the field subsample. They are strongly asymmetric, with off-centered bars with respect to the outer isophotes. One arm harbors a very bright complex of star formation not far from its connection to the bar (this complex is clearly delineated in our mid-infrared images) and the second arm is embryonic. In some magellanic barred spirals, the bar center does not correspond to the kinematic center (but this is not the case for NGC 4027, according to Pence et al. 1988). This morphology is thought to be driven by tidal interaction (Odewahn 1994), or can result from the cooperation between spiral waves of modes $m=1$ and $m=2$ (Tagger \& Athanassoula 1991), the two interpretations not being exclusive.

\subsection{Virgo subsample}

This subsample includes 9 or 10 spirals with normal HI content and 14 or $13 \mathrm{HI}$-deficient spirals (NGC 4293 is at the limit between the two categories). Hi-deficient galaxies do not necessarily belong to the cluster core, but can be found at large projected distances from M 87. For instance, NGC 4394, 4450, 4498 and 4580, with high Hi deficiencies, are located in the cluster periphery, between 1.3 and $2 \mathrm{Mpc}$ in projected distance from M 87.

Seven Virgo members of the sample have low blue or H-band luminosities ( $\mathrm{H}$ data from Boselli et al. 1997, used because they give an indication on the total stellar mass), and their sizes are also intrisically small. They are either normal in their Hi content (NGC 4351, 4430 and 4633) and of rather irregular morphology, except NGC 4634 seen edge-on, or deficient with no obvious distorsion (NGC 4413, 4491, 4498 and 4506).

Interestingly, the galaxies presented in this paper which have a central $F_{15} / F_{7}$ ratio above 2 are all Virgo spirals (except NGC 1022), with large Hi deficiencies. We computed the Hi deficiency according to Guiderdoni \& Rocca (1985) for the galaxies in our sample (the values are given in Paper I). This quantity is defined as the logarithmic difference in Hi mass surface density between a reference field galaxy sample and the galaxy considered, normalized by the dispersion in the field sample. We have adopted as the threshold for Hi dearth Def $>1.2$ (Paper I). For all the galaxies with a central color $F_{15} / F_{7}>2$ except NGC 1022 (namely NG 4569, 4293, 4388 and 4491), $1.2<D e f<2$. This suggests that the interaction with the intracluster gas also has consequences for the internal dynamics and reinforces the effects of the bar. This is not a systematic effect, since the remaining $10 \mathrm{HI}$-deficient galaxies have low to moderate central colors.

\section{Optical and mid-infrared disk sizes}

We have determined the size of the infrared emitting disk as explained at the beginning of Sect. 5, and also quantified the Hi deficiency, which is closely related to the anemia phenomenon (star formation being inhibited in parts of the outer disk where the gas density has dropped due to stripping). We find that the ratio of the infrared diameter, $D_{5}^{7} \mu \mathrm{Jy}$, to the optical diameter $D_{25}$, defined at 
the blue isophote $25 \mathrm{mag} \operatorname{arcsec}^{-2}$ (de Vaucouleurs et al. 1991), shows marked variations as a function of Hi deficiency (Fig. 4, top). Whereas $D_{5}^{7} \mu \mathrm{Jy} / D_{25}$ is of the order of 0.7-0.9 for gas-rich spirals (none of the Virgo galaxies belongs to this category) and 0.96 for NGC 4634 which is seen edge-on, it ranges between 0.5 and 0.8 for spirals of intermediate Hi content, and between 0.35 and 0.65 for deficient spirals, except 0.77 for NGC 4647 whose outskirts may be contaminated by the emission of the neighbor elliptical NGC 4649. The value 0.35 is that of NGC 4569, known for its spectacular anemia, and NGC 4438, which has undergone a violent collision (Combes et al. 1988). The only exception to this trend is NGC 289, which is very rich in Hi but has a ratio $D_{5}^{7} \mu \mathrm{Jy} / D_{25}$ of about 0.4 . We again insist that its outer disk is of very low surface brightness, and that the condition for star formation in such disks is
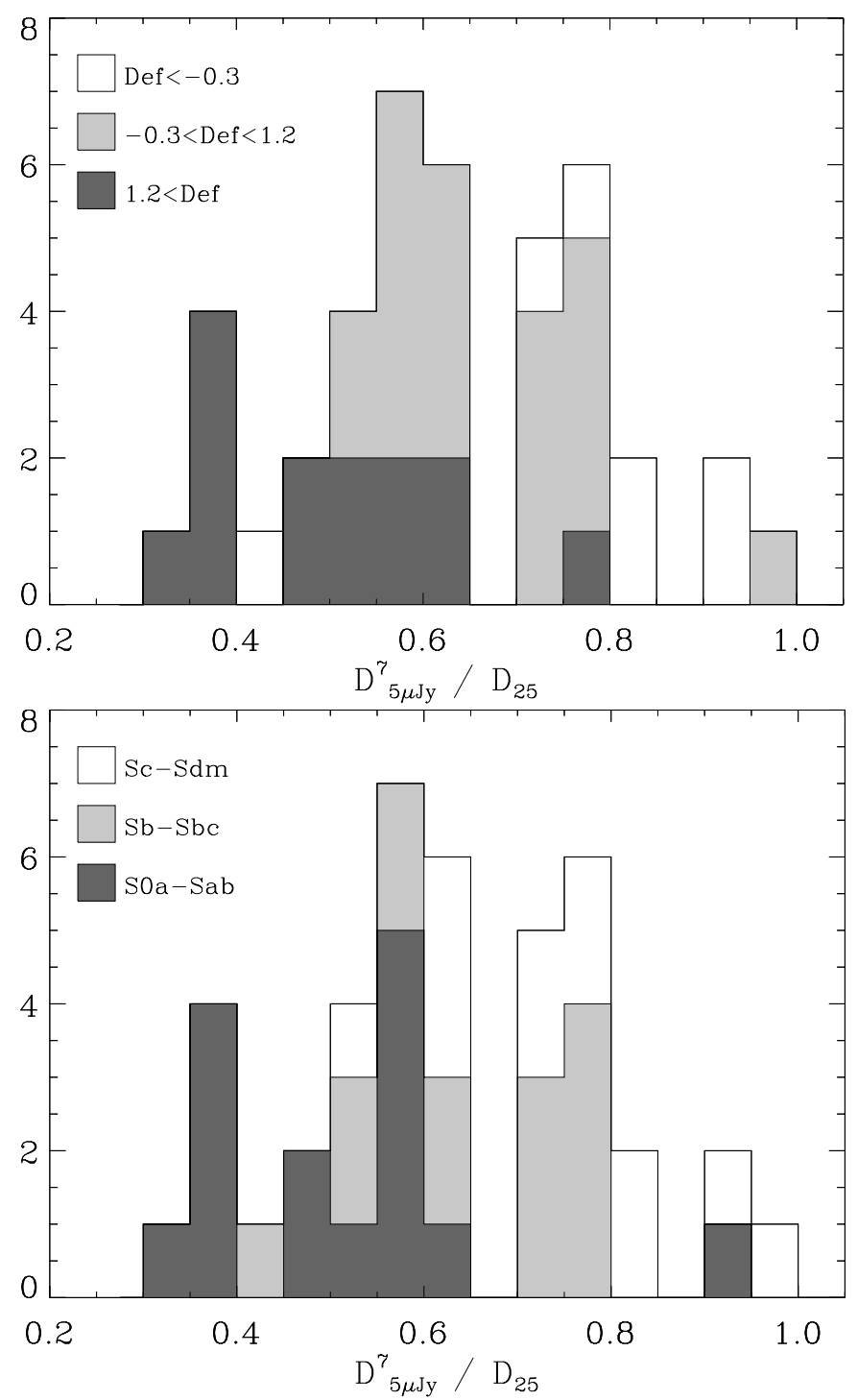

Fig. 4. Histograms of mid-infrared to optical size ratios as a function of HI-deficiency and morphological type. Def $>1.2$ corresponds to severely HI-stripped galaxies and Def $<-0.3$ to gas-rich galaxies likely to be very different from those in ordinary bright disk galaxies.

There is also a trend for $D_{5}^{7} \mu \mathrm{Jy} / D_{25}$ to be lower in early-type than in late-type spirals, as shown in Fig. 4 (bottom). However, this relationship is not independent of the first one, since HI-deficient galaxies are mostly classified as early, due to the dominance in practice of the criterion based on the aspect of the arms in the Hubblesystem classification (see Paper I). Early-type spirals are essentially characterized by smooth and faint outer disks, whereas in latest types, the arms are bright and patchy in their whole length, structured by star formation sites. Hence, a natural interpretation of both histograms shown here is that the ratio of mid-infrared to optical sizes primarily depends on the star formation activity in outer disks, which is strongly coupled to the gas density.

\section{Summary}

We have presented complete maps of dust emission at $7 \mu \mathrm{m}$ of 43 galaxies spanning the spiral sequence from types $\mathrm{S} 0 /$ a to $\mathrm{Sdm}$, at an angular resolution less than $10^{\prime \prime}$ and a sensitivity of the order of $5 \mu \mathrm{Jy} \operatorname{arcsec}^{-2}$. We have also detailed the data reduction, provided the total fluxes at 7 and $15 \mu \mathrm{m}$ and outlined the generic morphological properties in this sample. Since there exists an abundant literature on many of these galaxies studied individually, the present atlas has concentrated on common features. The detailed description of the sample and interpretation of the data are given together in Paper I.

The morphology is very similar in the mid-infrared and in the optical, except in peculiar galaxies where the dust emission is highly concentrated: the 7 and $15 \mu \mathrm{m}$ emission follows tightly spiral arms, lenses and individual giant complexes. It appears however less extended than the stellar emission and tends to be reduced in smooth and unstructured spiral arms often seen in the outer disks of early-type galaxies and especially of $\mathrm{HI}$-deficient galaxies. This can be related to low densities of both the gas and the young stellar population. Another noticeable difference concerns the aspect of the bar, where the responses of the interstellar tracers and of the stellar component to the gravitational potential are clearly distinct. Finally, central regions stand out in our maps as entities with particular properties, and are studied in detail in Paper I.

The major characteristic of mid-infrared colors, i.e. $15 \mu \mathrm{m}$ to $7 \mu \mathrm{m}$ flux ratios, is that they are remarkably uniform throughout disks, except in some circumnuclear regions and a few bright complexes in spiral arms (the best example of the latter can be found in the outer interacting arms of M 101).

The maps will be made available to the community, in fits format, in the ISO public archive (http://www.iso.vilspa.esa.es/). They can be used for studies of the interplay between dust and other components of galaxies, and constitute the basis for two following papers. 

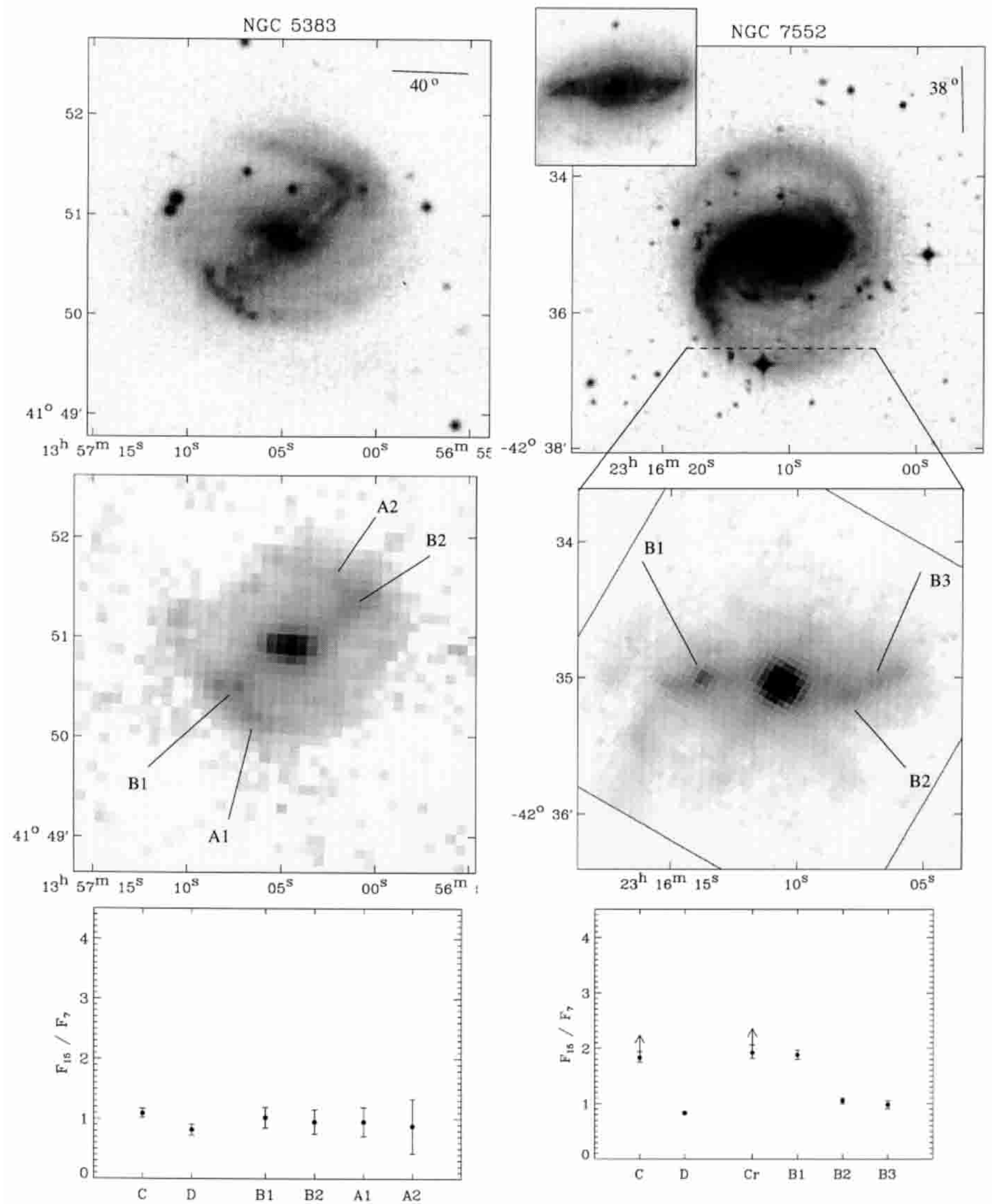

Fig. 5. Strongly barred galaxies in order of decreasing bar strength. a) NGC 5383 and 7552 , with $D_{\text {bar }} / D_{25} \approx 0.65$ and 0.67 . In all figures are displayed, from top to bottom: an optical image from the DSS with, in a corner, a segment showing the orientation of the line of nodes and the inclination value; the $7 \mu \mathrm{m}$ map; $F_{15} / F_{7}$ colors of selected regions ("C": circumnuclear region; "D": averaged disk; "Cr": central resolution element). Locations in the bar ("B") or the arms ("A") are indicated on the $7 \mu \mathrm{m}$ map, which is scaled identically to the optical map except when a dashed segment outlines the field of view shown below 

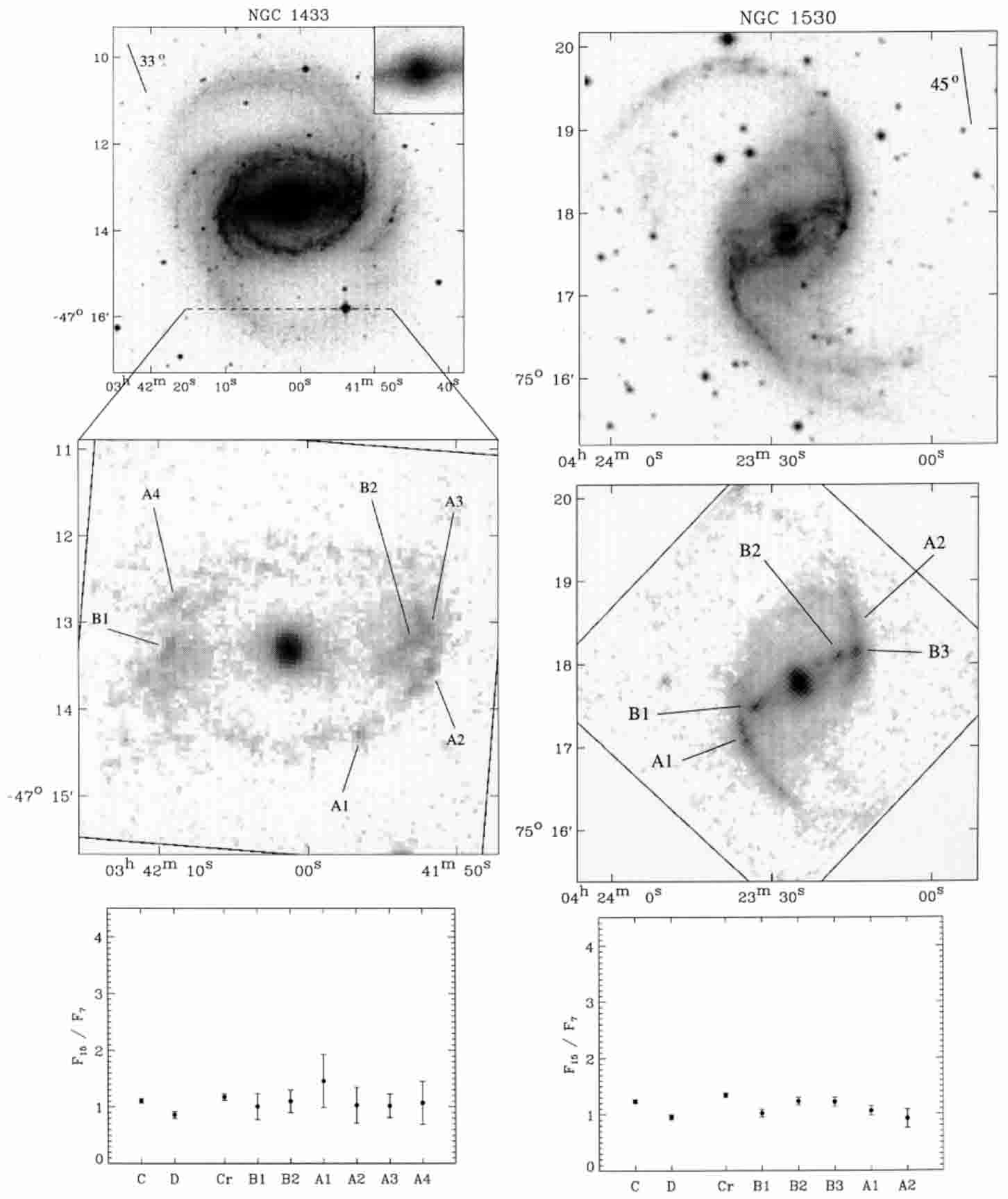

Fig. 5. b) NGC 1433 and 1530 , with $D_{\text {bar }} / D_{25} \approx 0.54$ and 0.48 

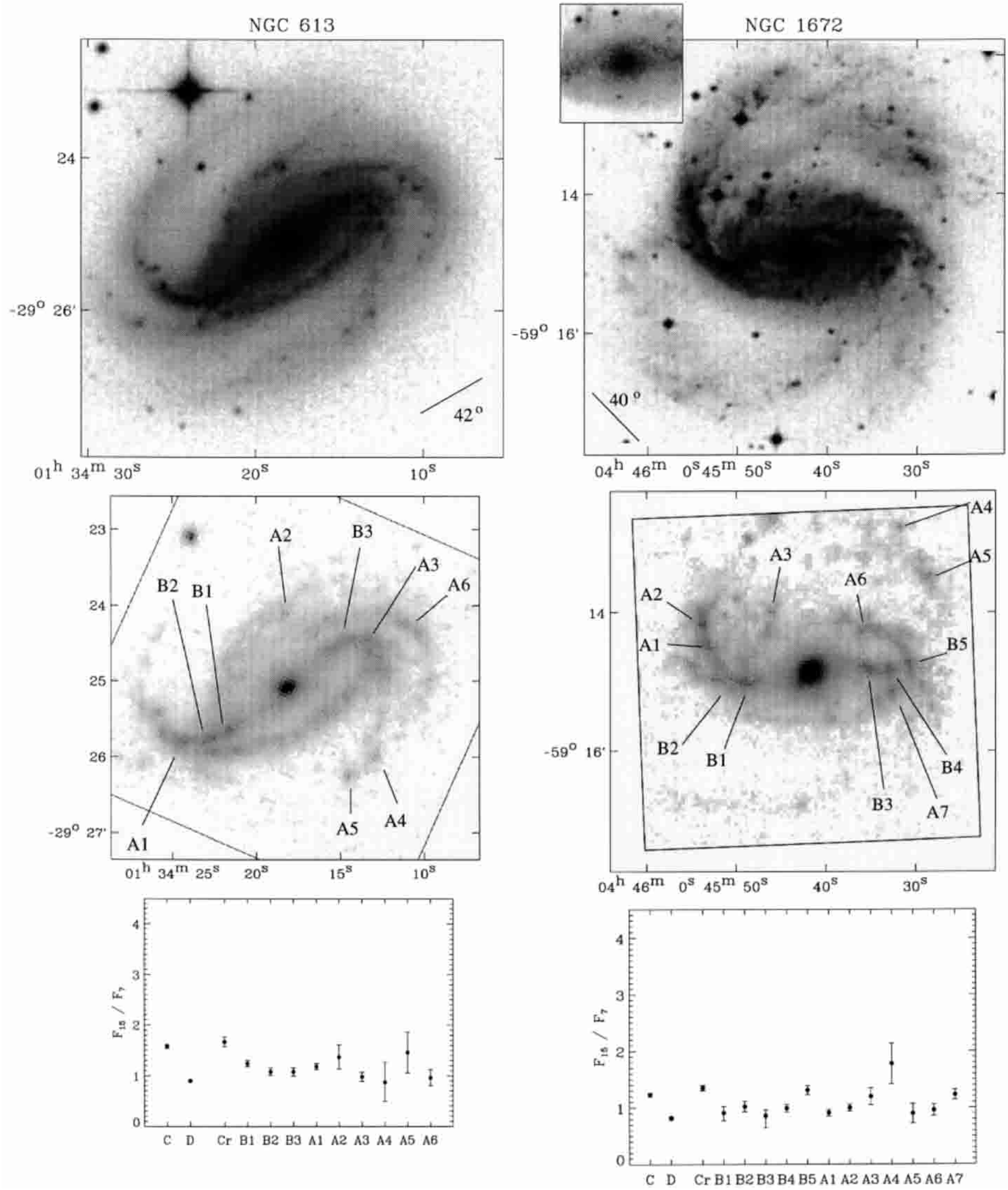

Fig. 5. c) NGC 613 and 1672 , with $D_{\text {bar }} / D_{25} \approx 0.45$ and 0.42 

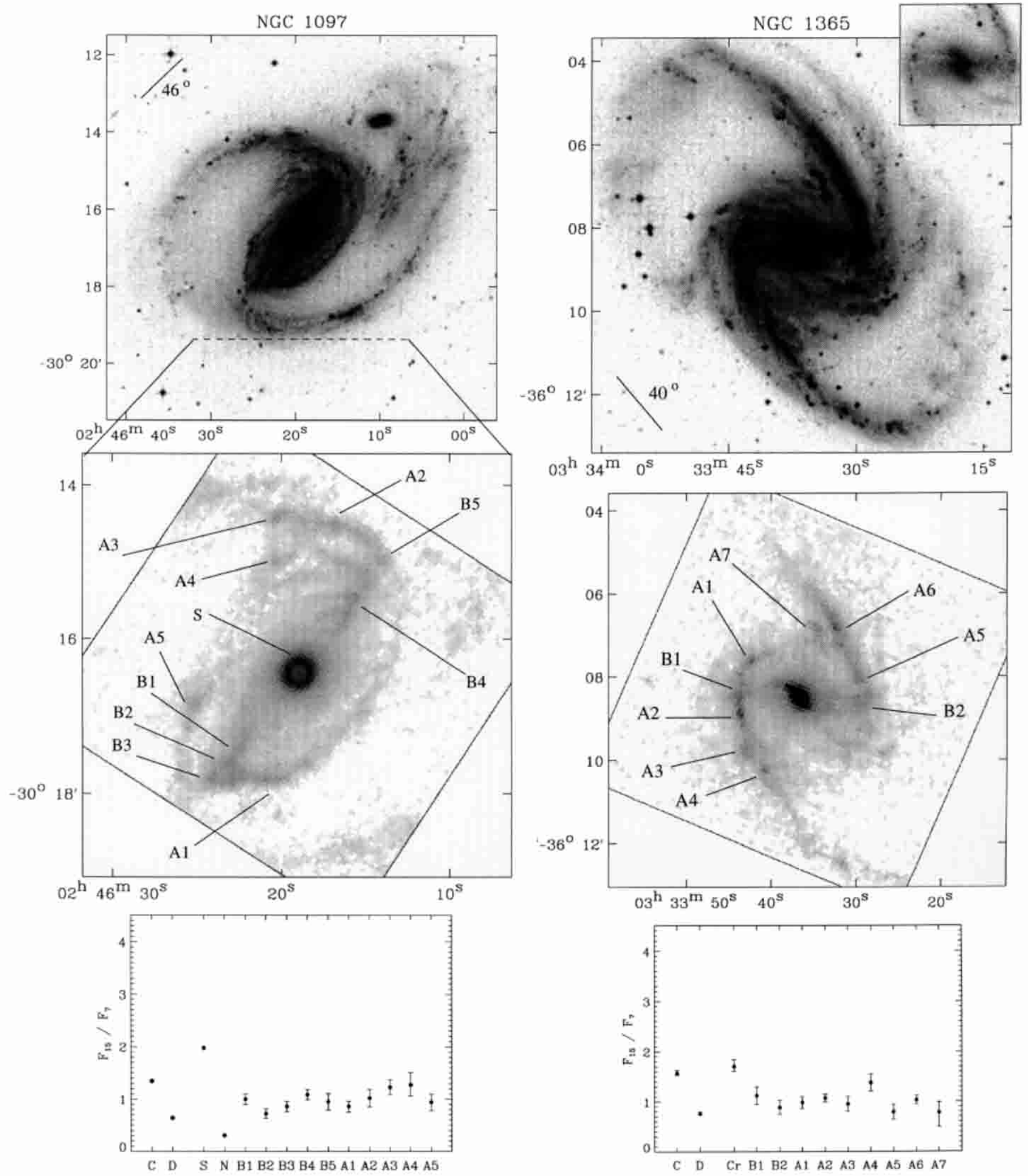

Fig. 5. d) NGC 1097 and 1365 , with $D_{\text {bar }} / D_{25} \approx 0.35$ and 0.31 . The labels "S" and "N" correspond to the brightest spot in the ring and the nucleus, whose colors were estimated from the images treated with the analog of CLEAN (Sect. 3.4), with no error bars 

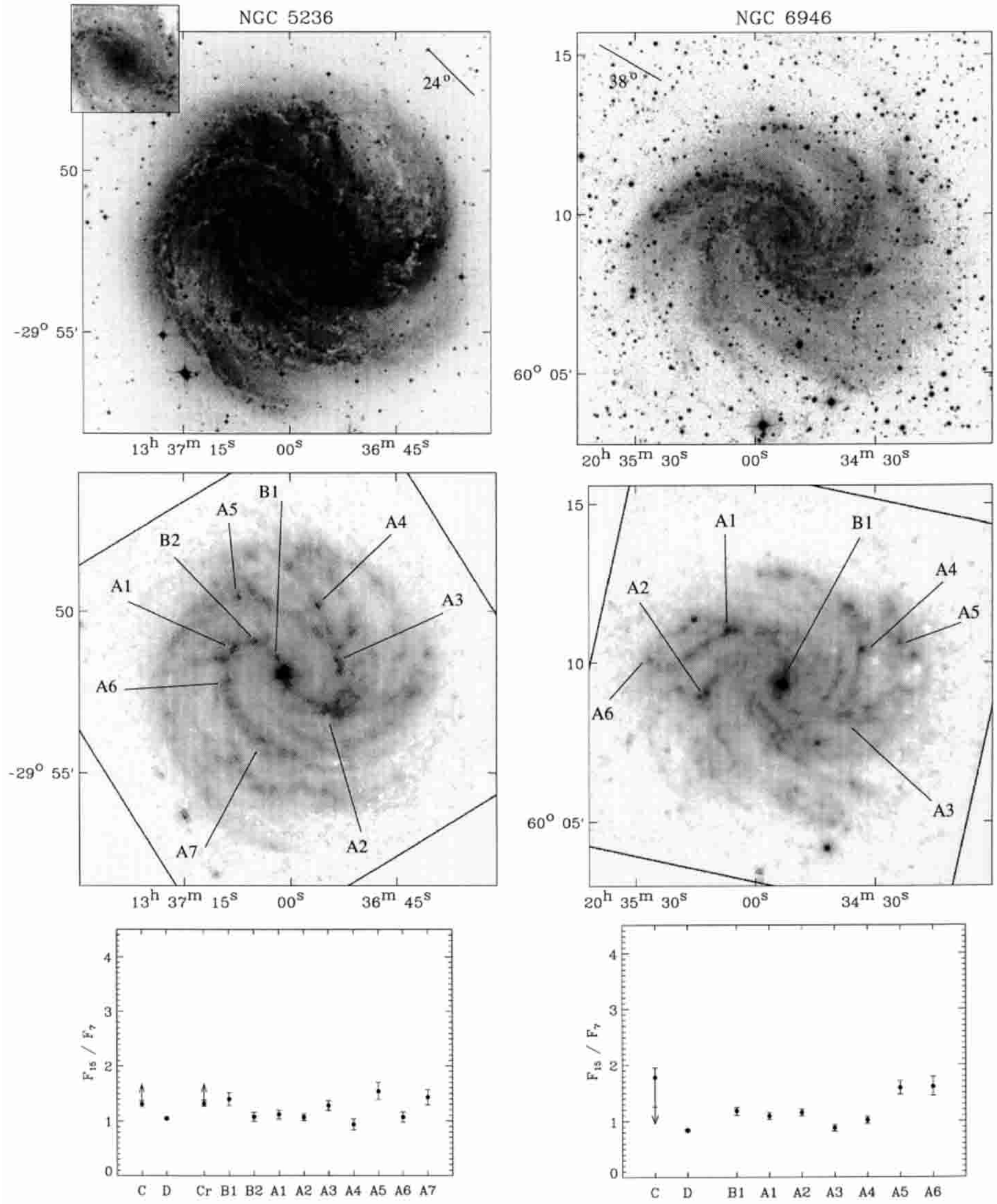

Fig. 6. Weakly barred and unbarred galaxies. a) NGC 5236 and 6946 , moderately barred with $D_{\text {bar }} / D_{25} \approx 0.24$ and 0.19 


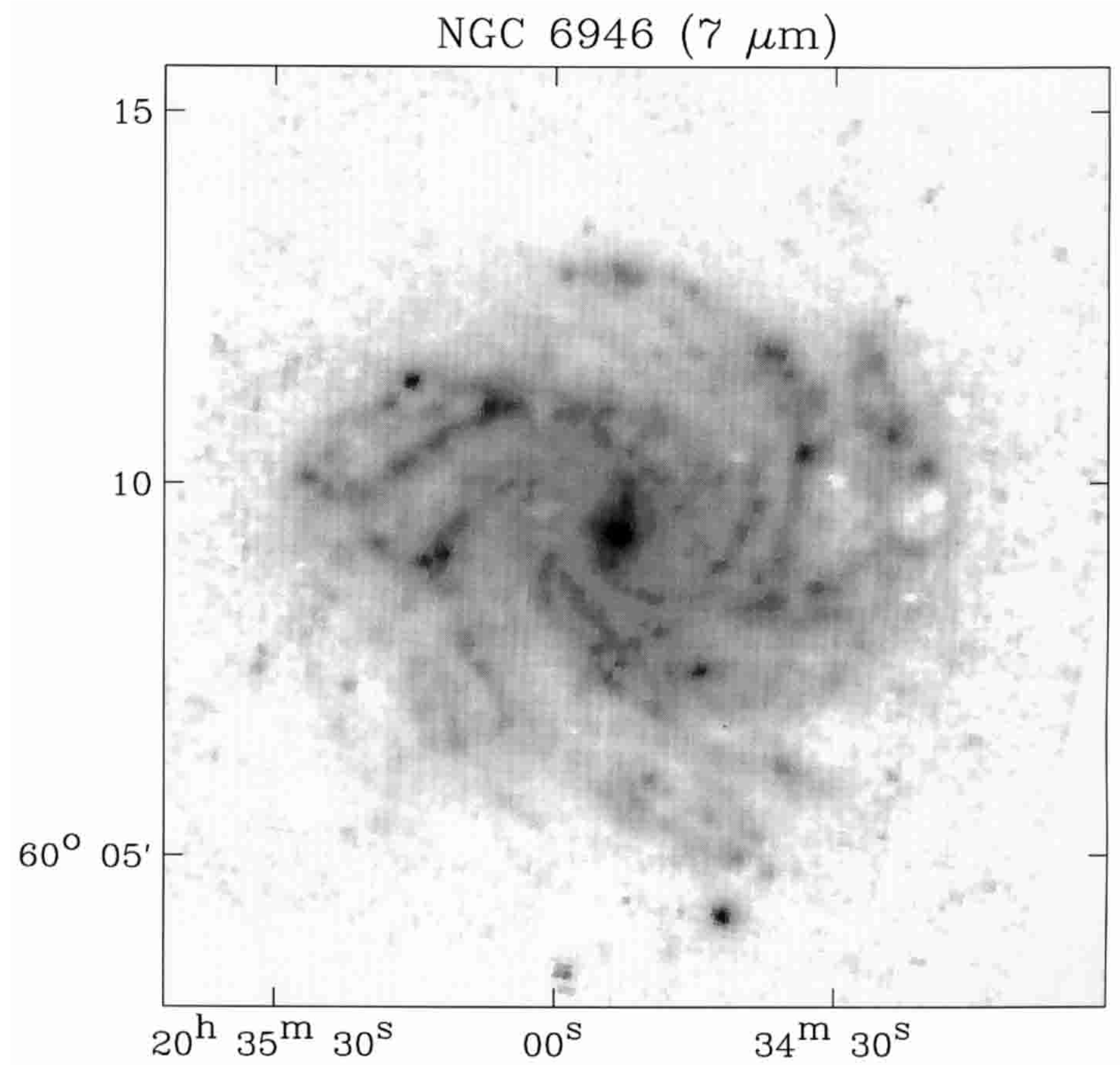

Fig. 6. a') Enlargement of the $7 \mu \mathrm{m}$ map of NGC 6946 

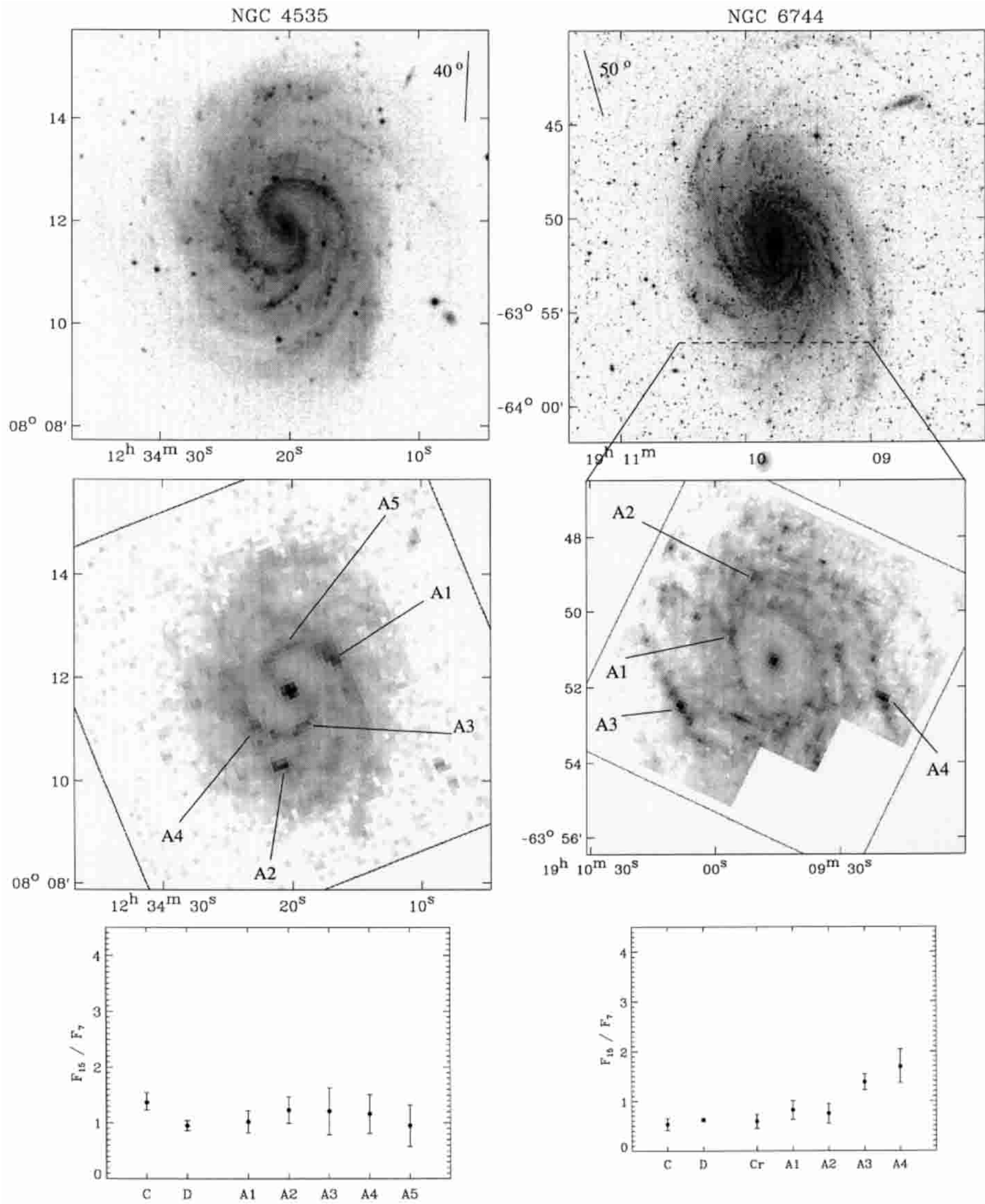

Fig. 6. b) NGC 4535 and 6744 , weakly barred with $D_{\text {bar }} / D_{25} \approx 0.18$ and 0.17 

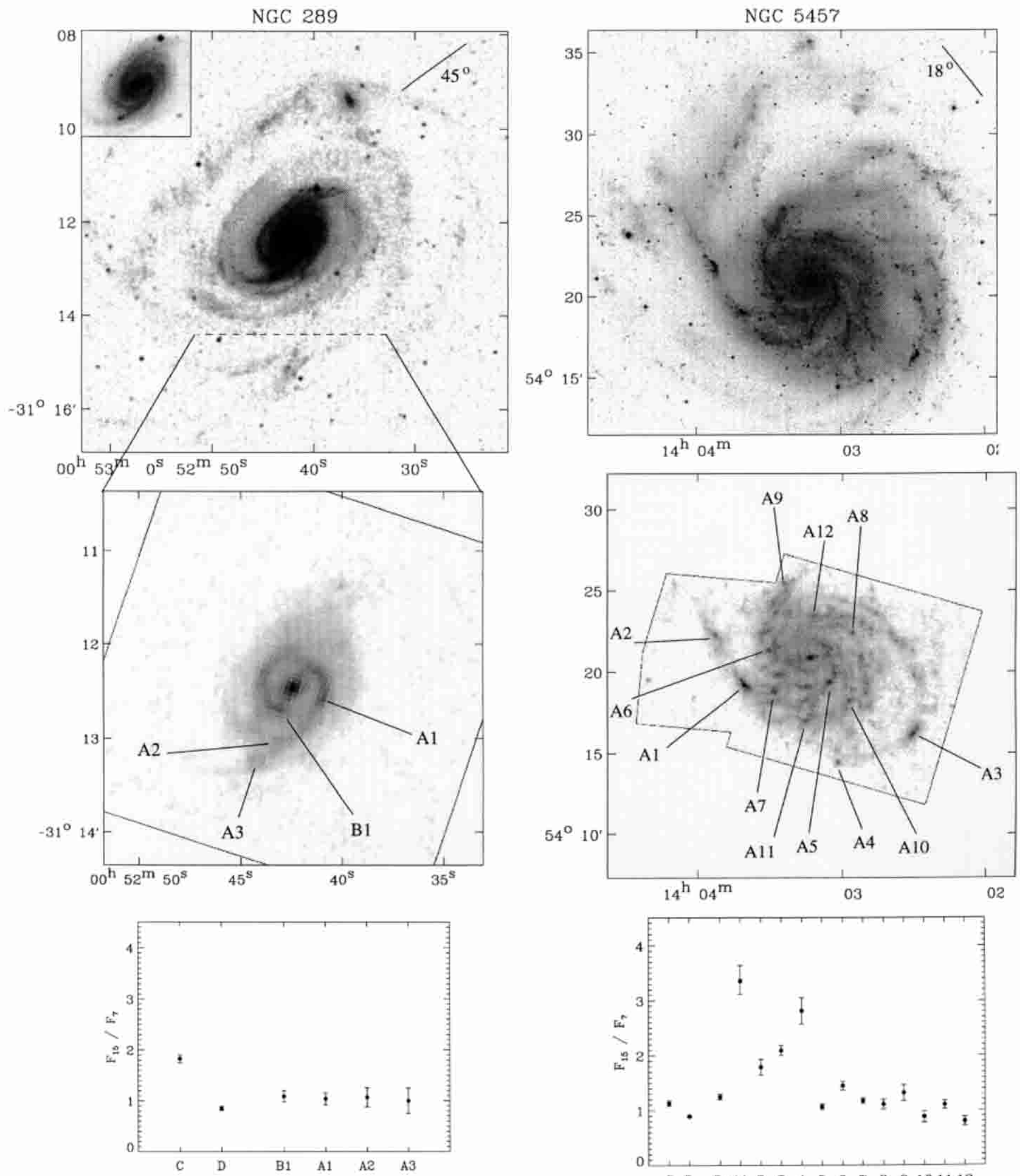

Fig. 6. c) NGC 289 and 5457 , with $D_{\text {bar }} / D_{25} \approx 0.15$ and 0.06

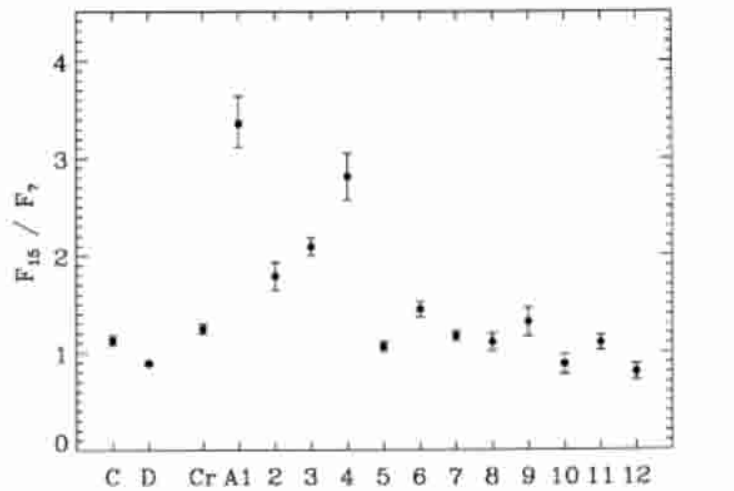




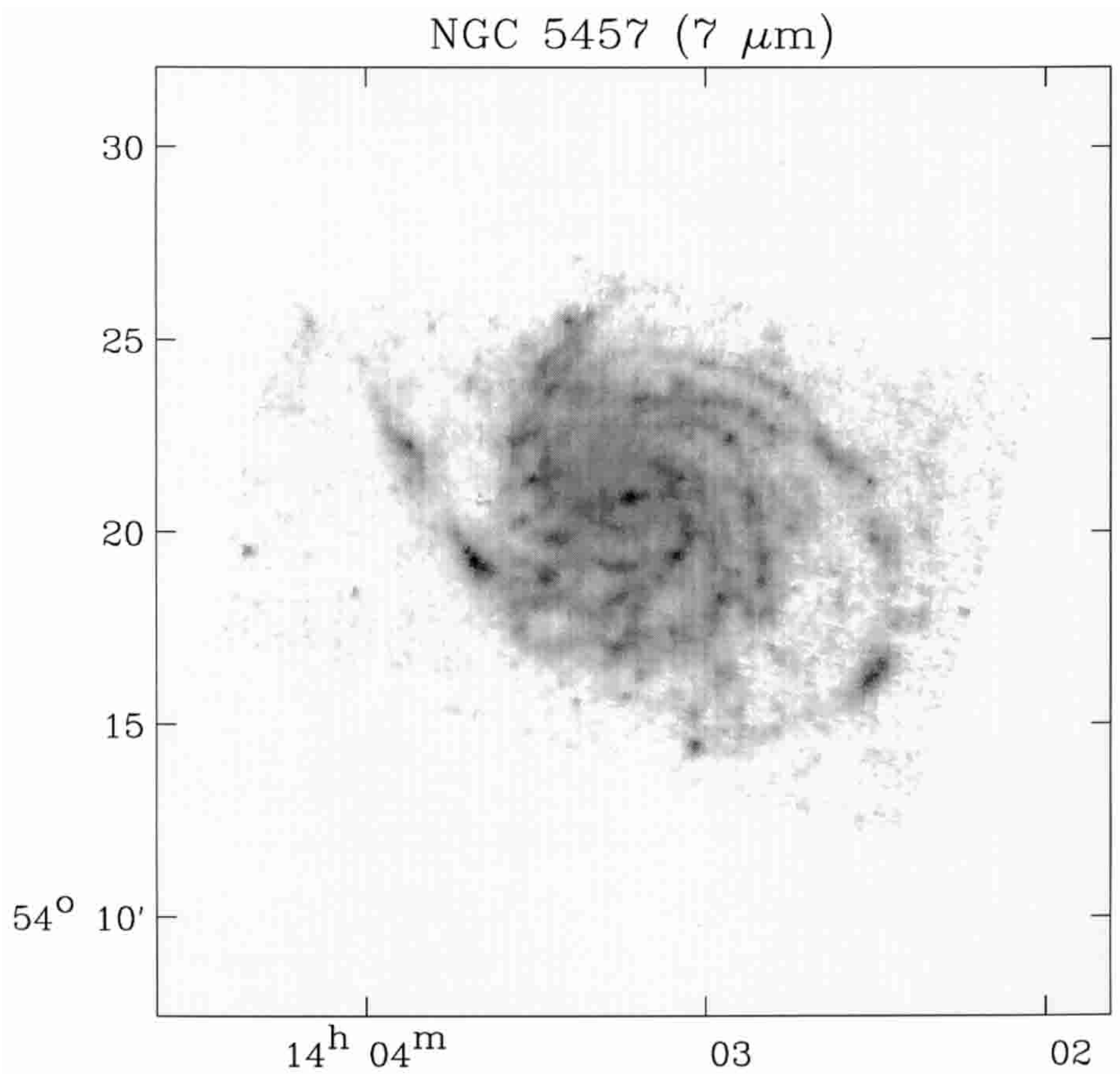

Fig. 6. c') Enlargement of the $7 \mu \mathrm{m}$ map of NGC 5457 

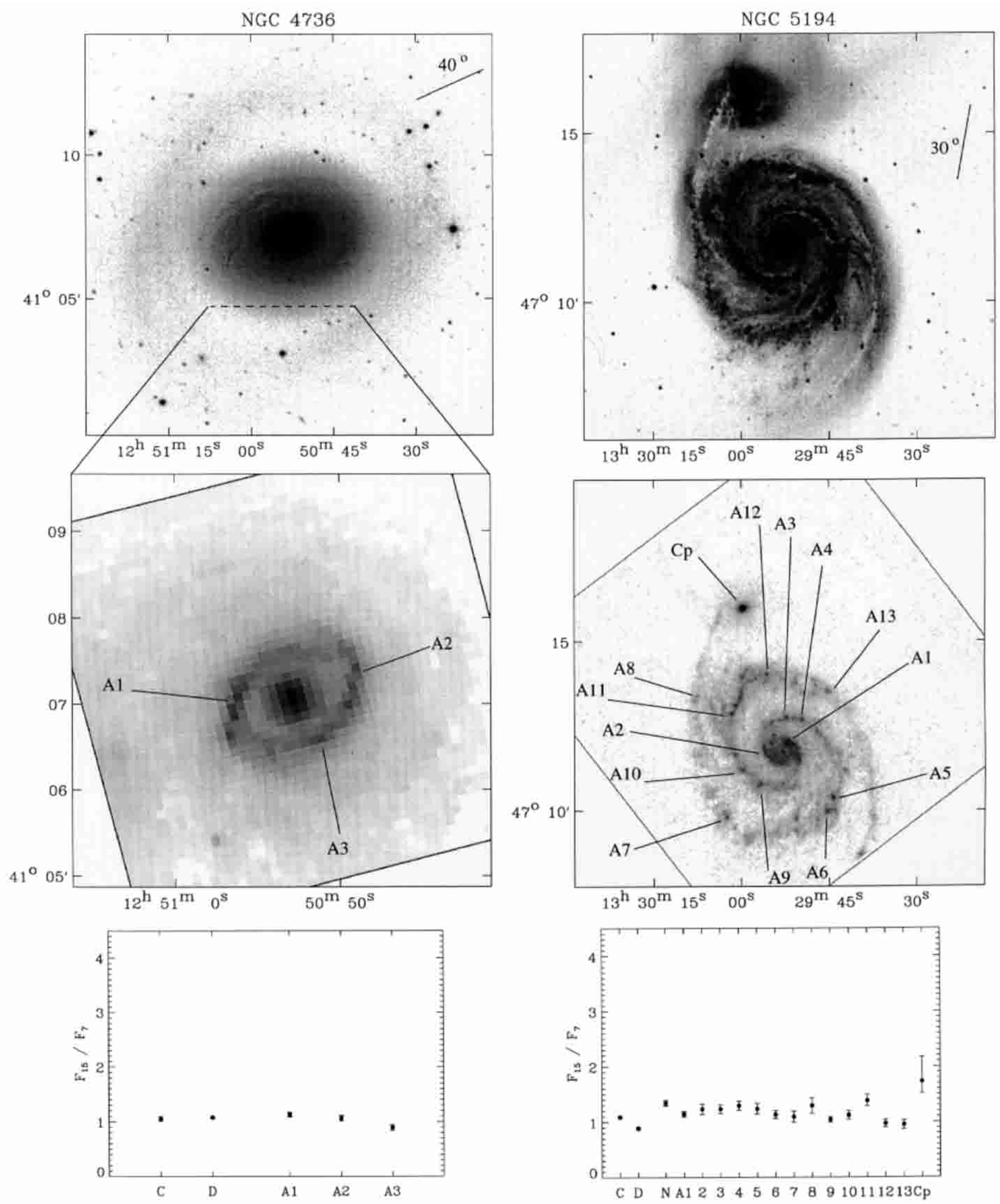

Fig. 6. d) NGC 4736 and 5194, unbarred galaxies (NGC 4736 possesses in fact an oval distorsion). The labels "N" and "Cp" refer to the nucleus of NGC 5194 and the nucleus of its companion, NGC 5195. Note that in NGC 5194, the thin NE and SW outermost parts of both arms seen in the mid-infrared coincide with dust lanes seen in absorption 


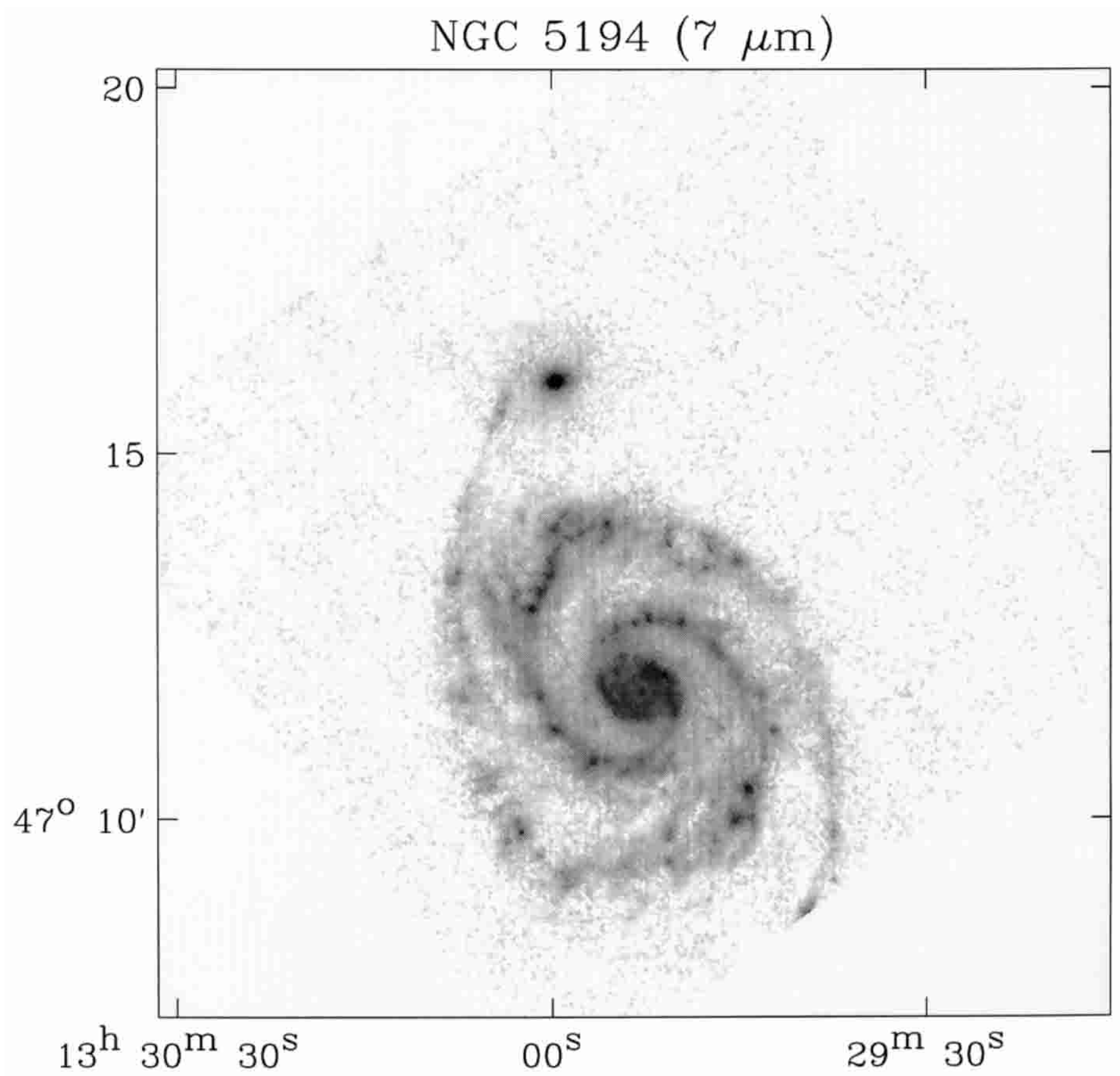

Fig. 6. d) Enlargement of the $7 \mu \mathrm{m}$ map of NGC 5194 

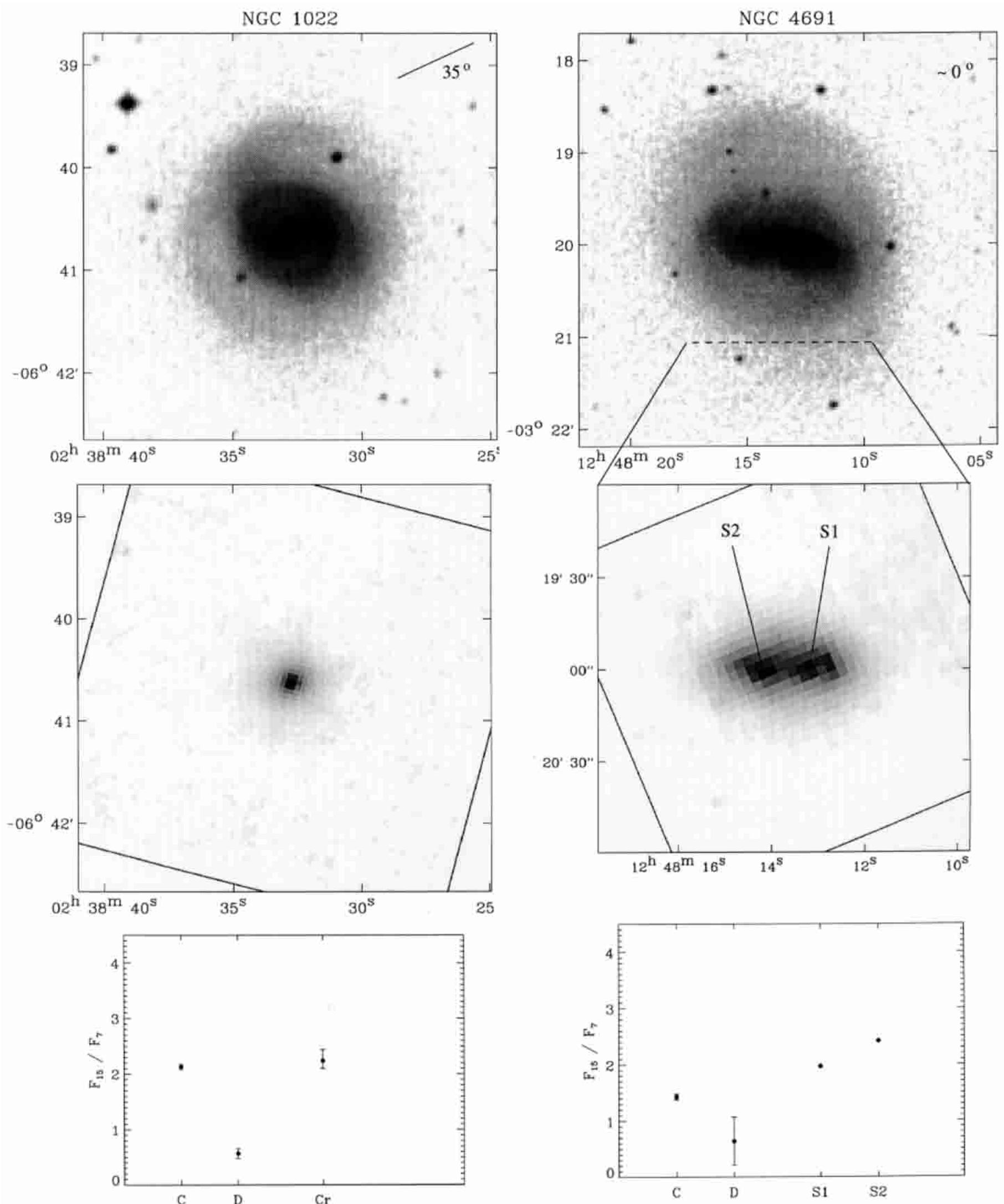

Fig. 7. Peculiar galaxies. a) NGC 1022 and 4691 , amorphous barred spirals (possibly merger results). They have $D_{\text {bar }} / D_{25} \approx 0.32$ and 0.43. The labels "S1" and "S2" refer to the two resolved spots in the center of NGC 4691, whose colors were estimated from the images treated with the analog of CLEAN, with no error bars 

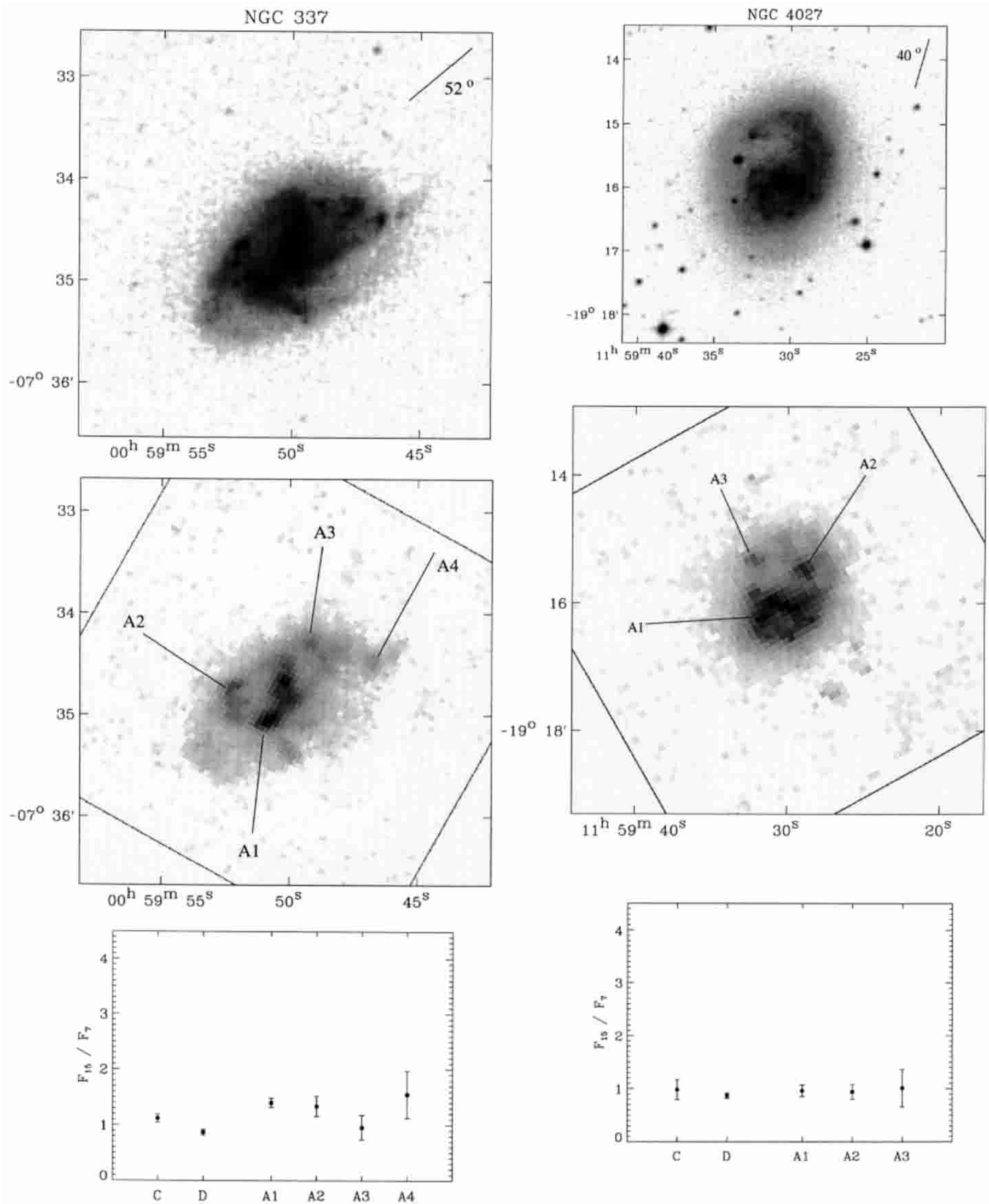

Fig. 7. b) NGC 337 and 4027, magellanic barred spirals. The bar length was not estimated because it is difficult to delineate the bar precisely 

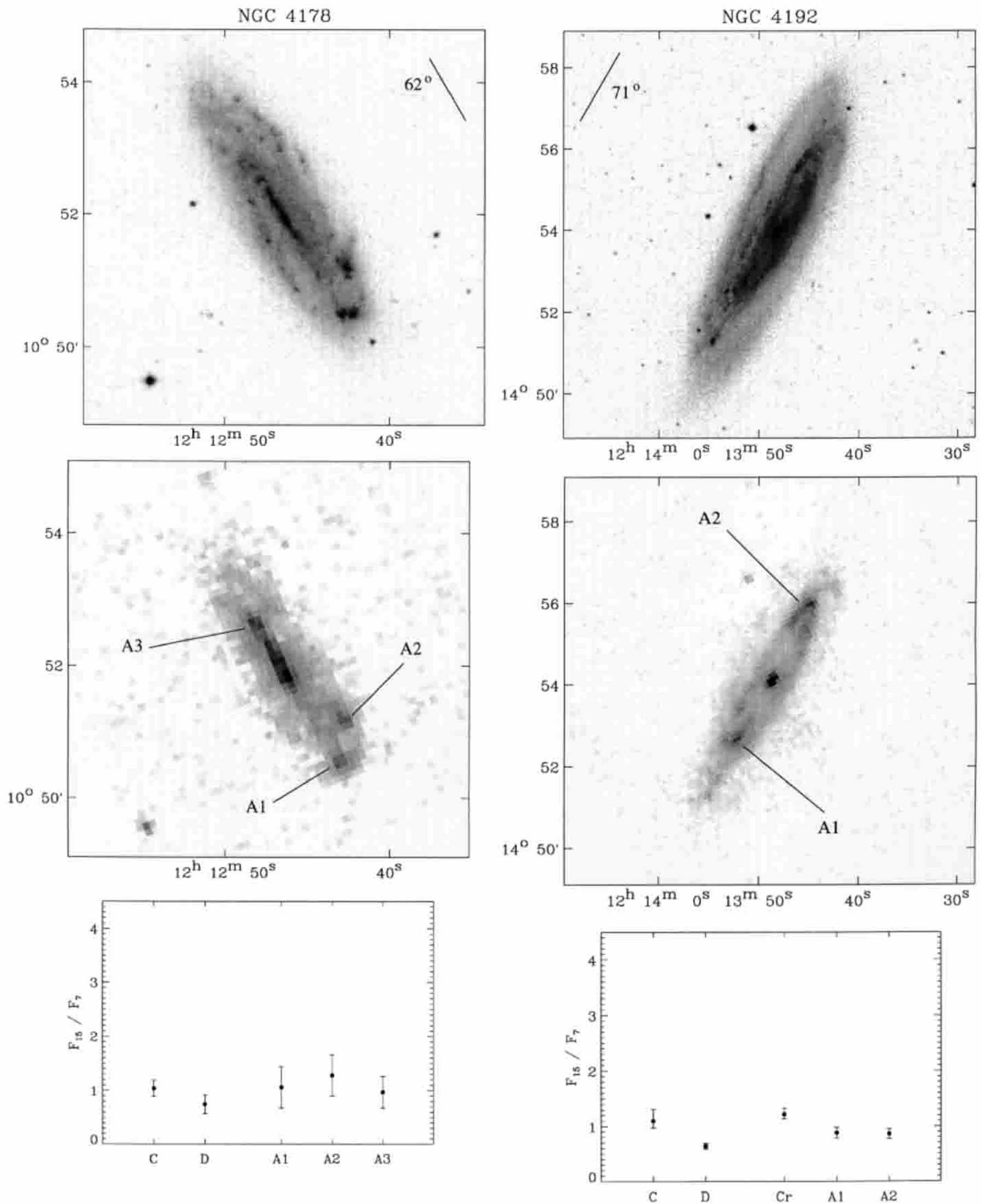

Fig. 8. Virgo galaxies which are not HI-deficient. a) NGC 4178 and 4192 

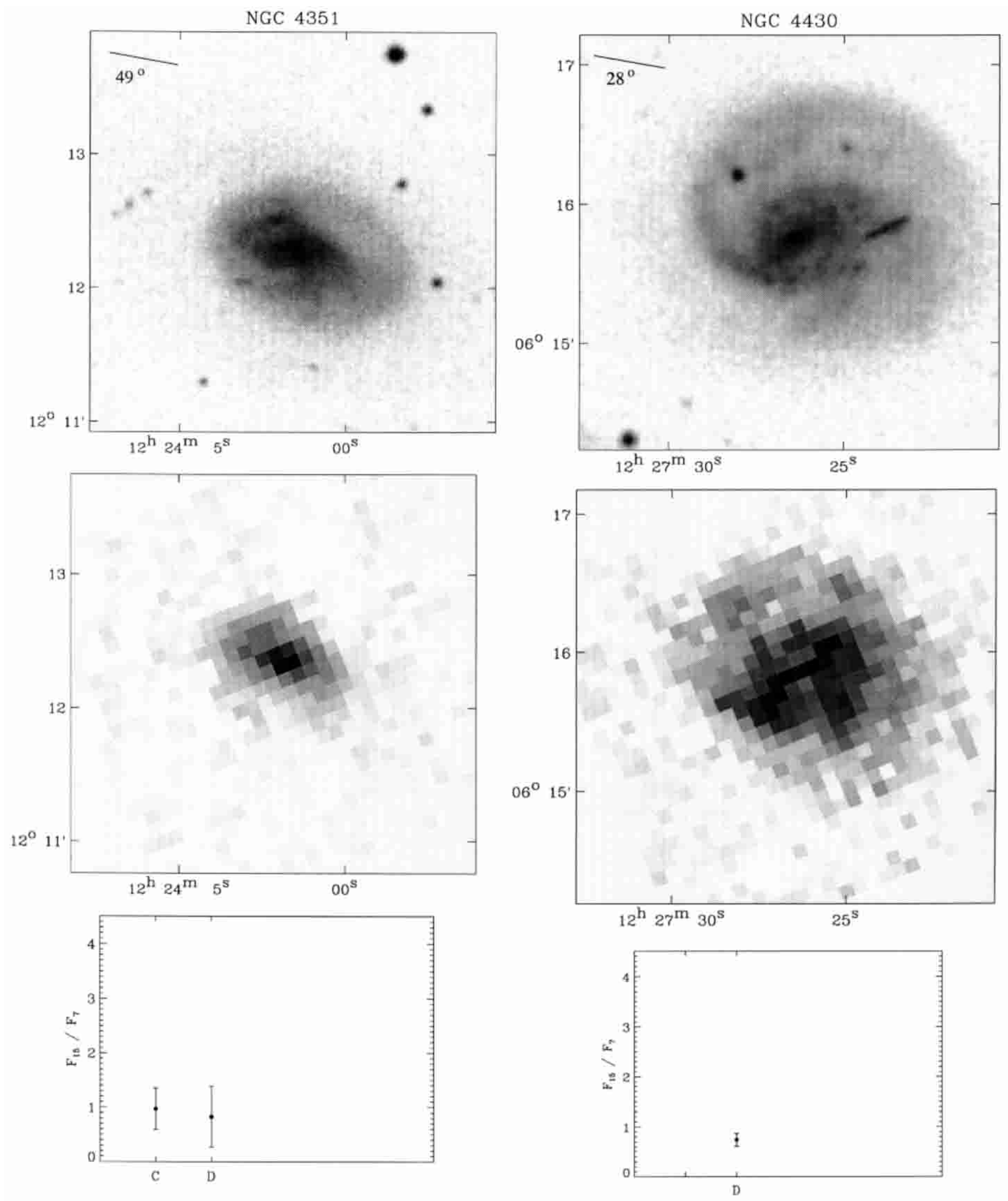

Fig. 8. b) NGC 4351 and 4430 

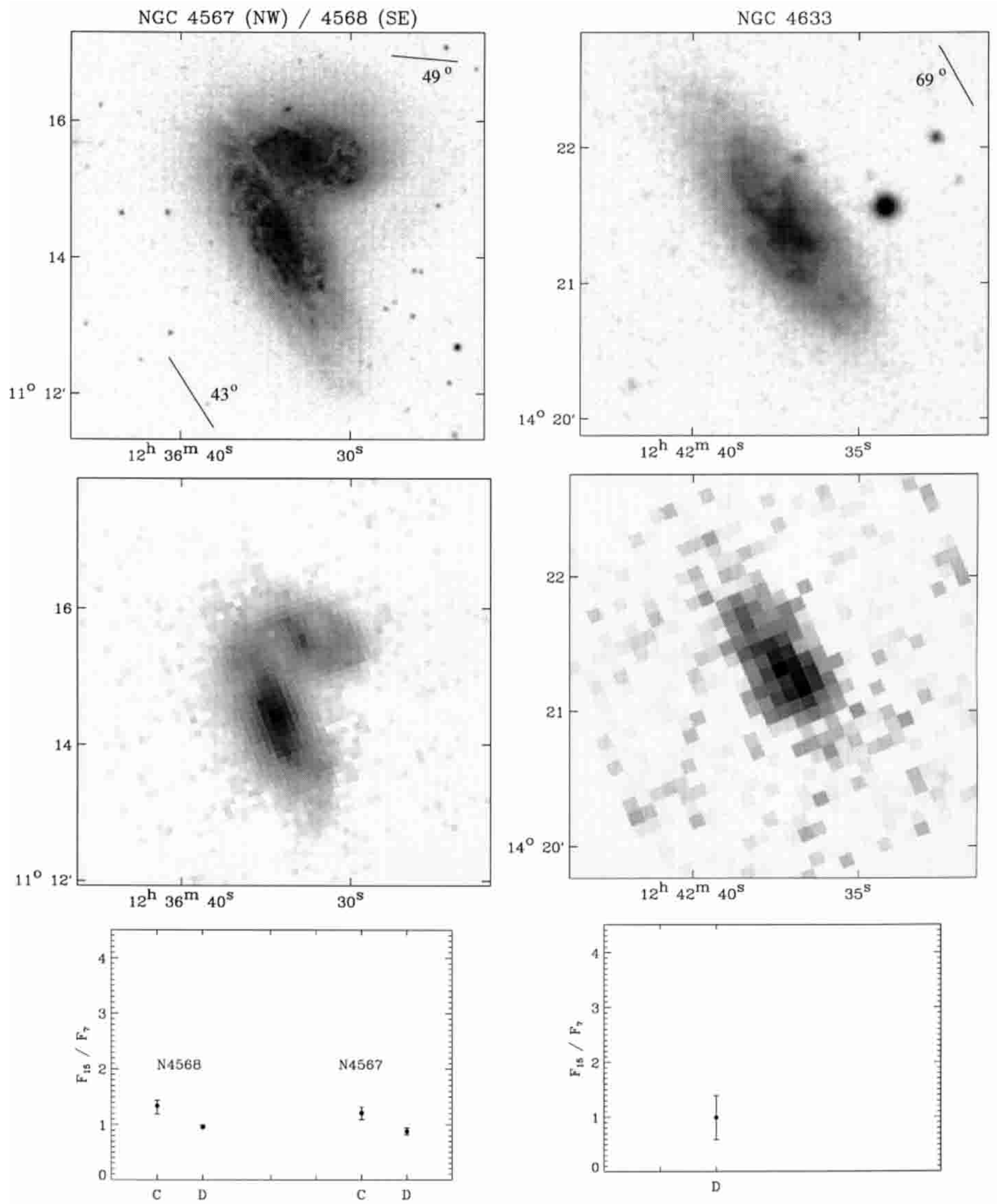

Fig. 8. c) NGC $4567 / 8$ and 4633 

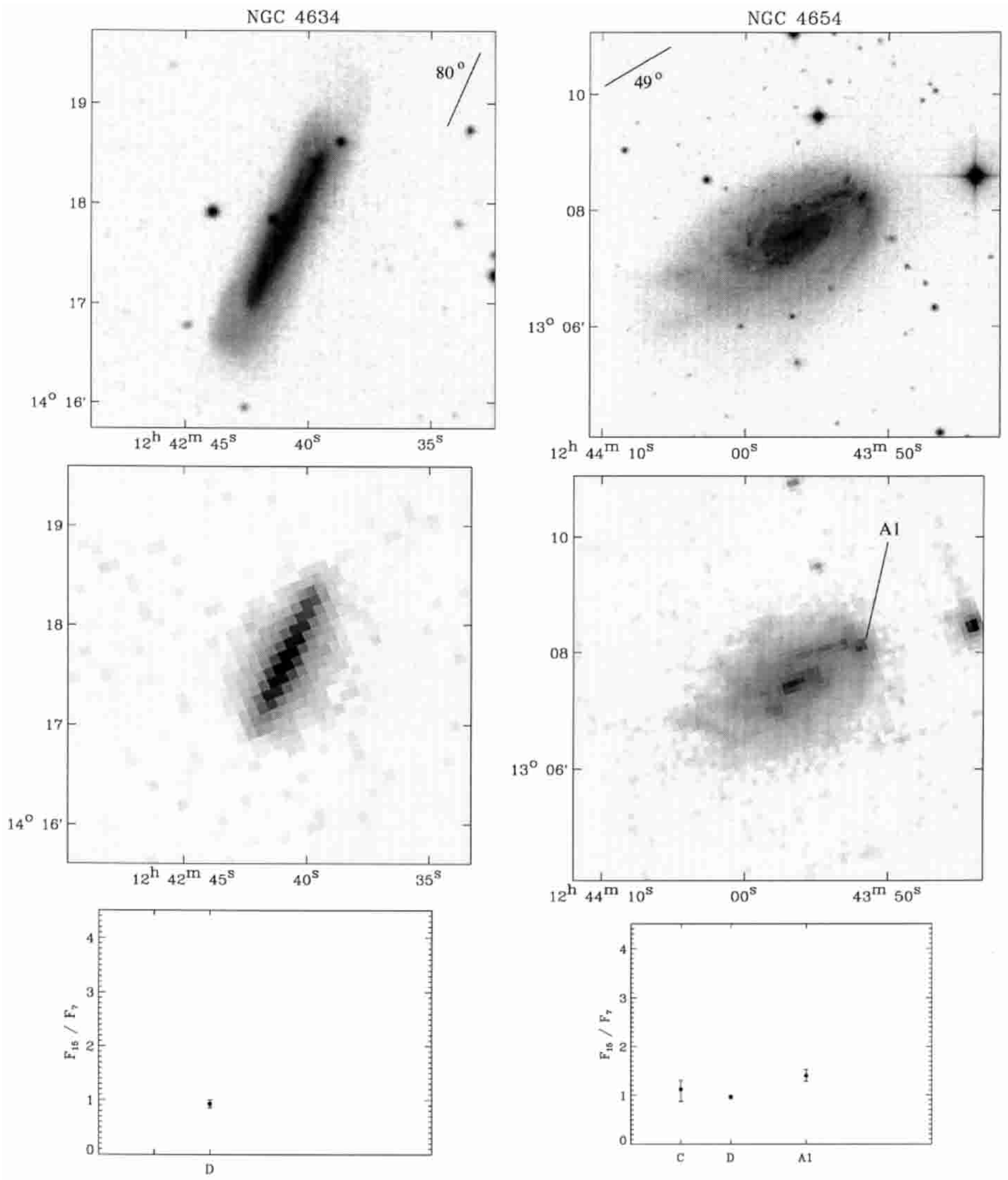

Fig. 8. d) NGC 4634 and 4654 

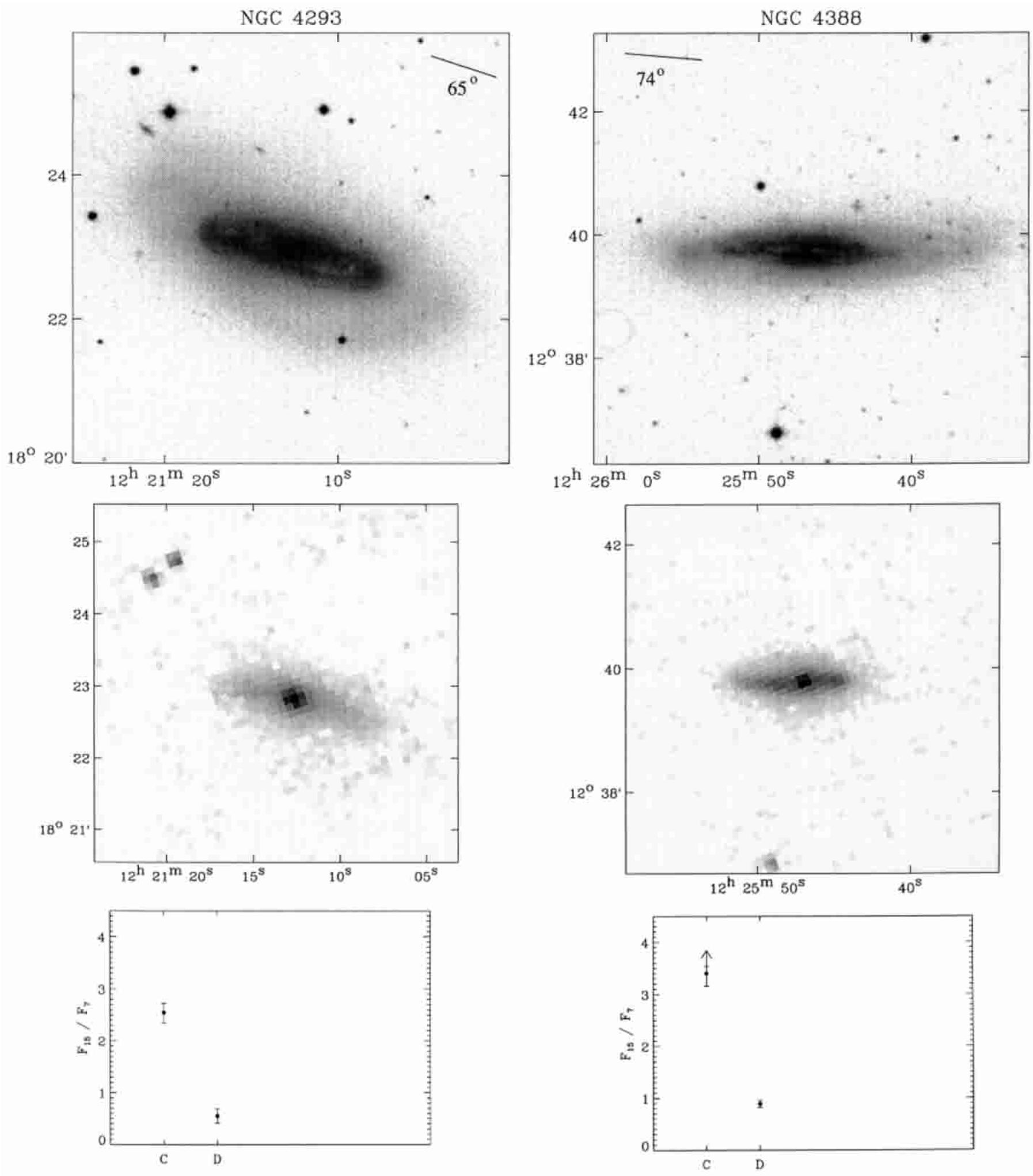

Fig. 9. HI-deficient Virgo galaxies. a) NGC 4293 and 4388 

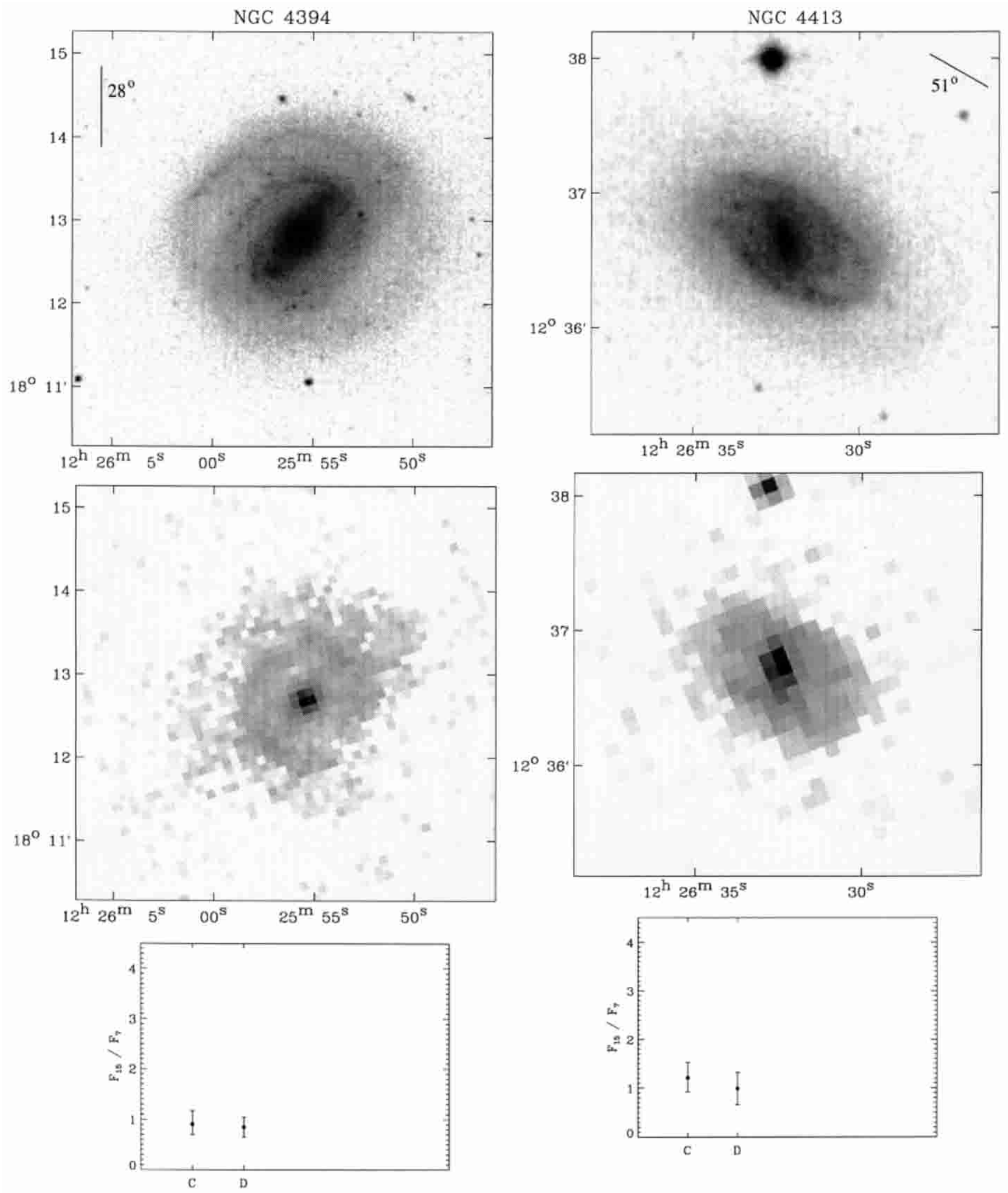

Fig. 9. b) NGC 4394 and 4413 

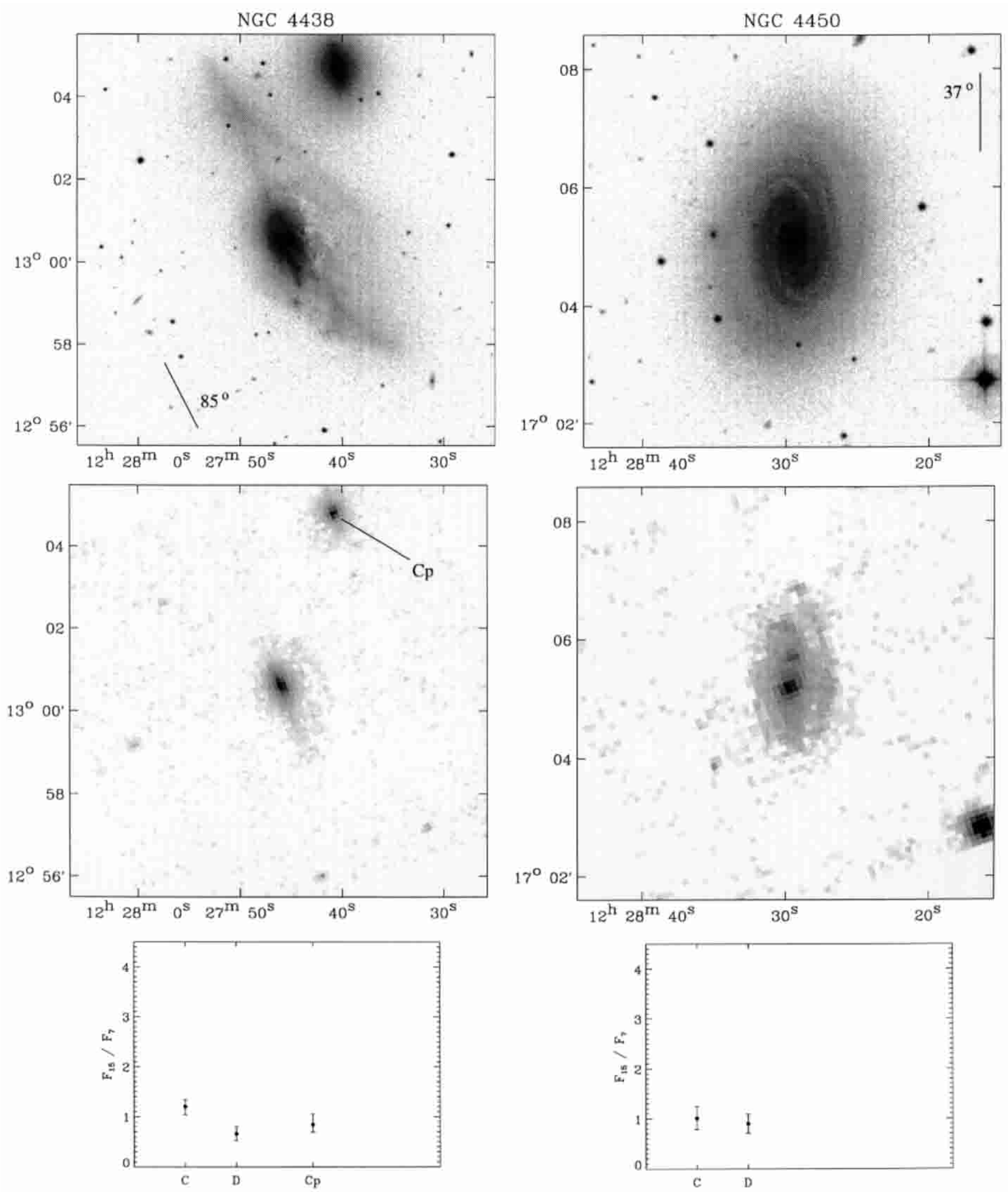

Fig. 9. c) NGC 4438 and 4450. The label "Cp" refers to the nucleus of NGC 4435, which is likely at the origin of the disruption of NGC 4438 (Combes et al. 1988) 

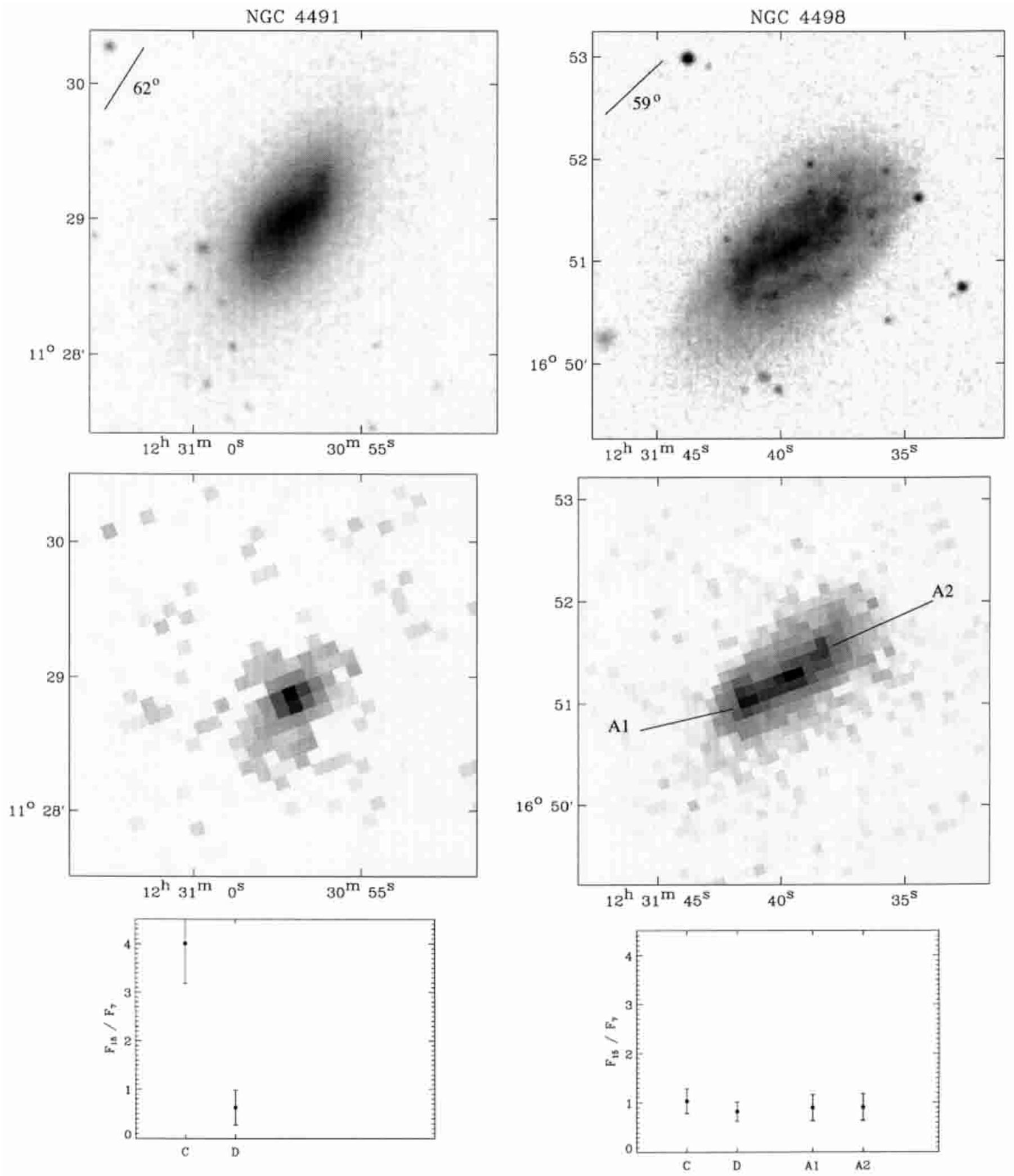

Fig. 9. d) NGC 4491 and 4498 

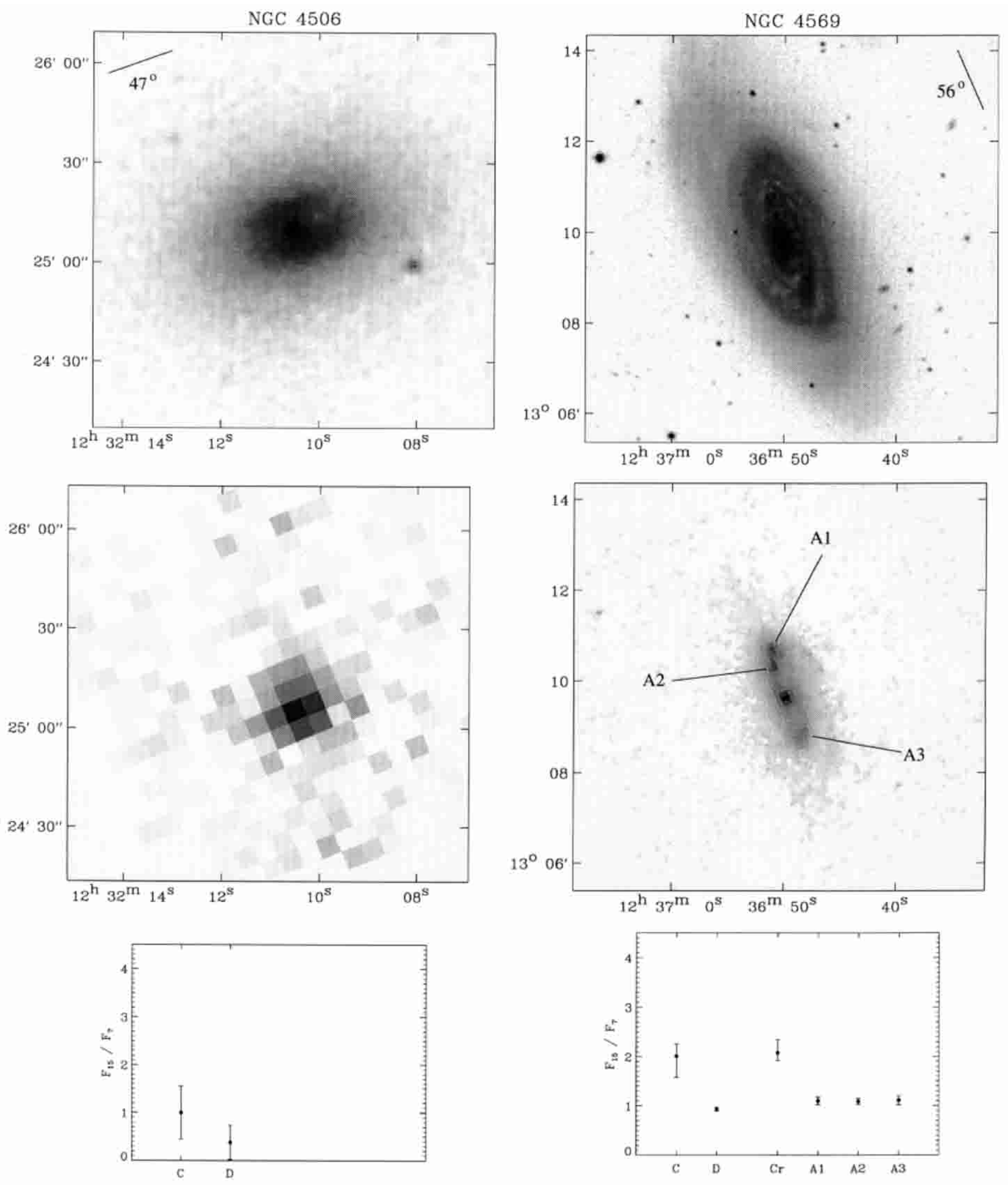

Fig. 9. e) NGC 4506 and 4569 

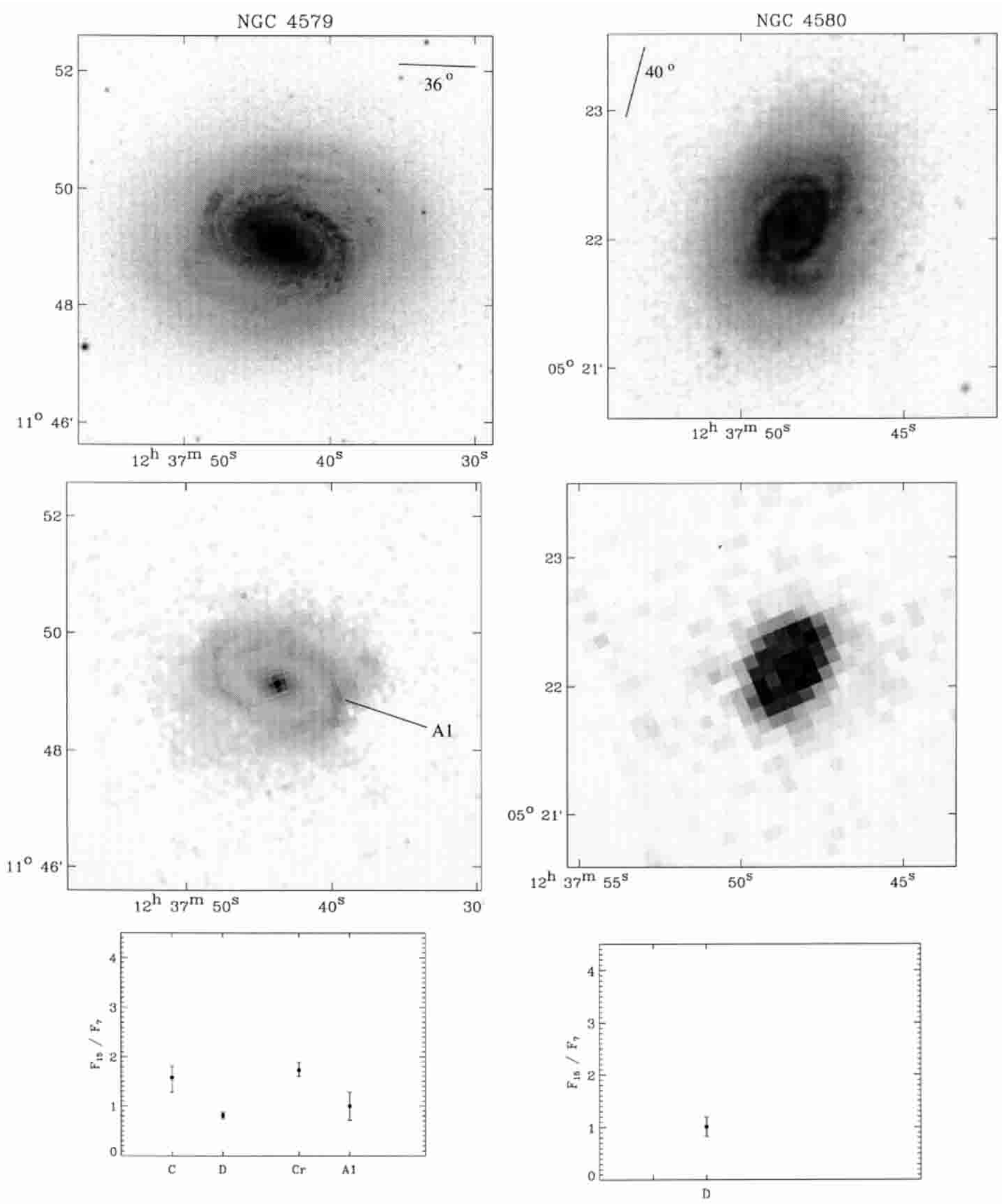

Fig. 9. f) NGC 4579 and 4580 

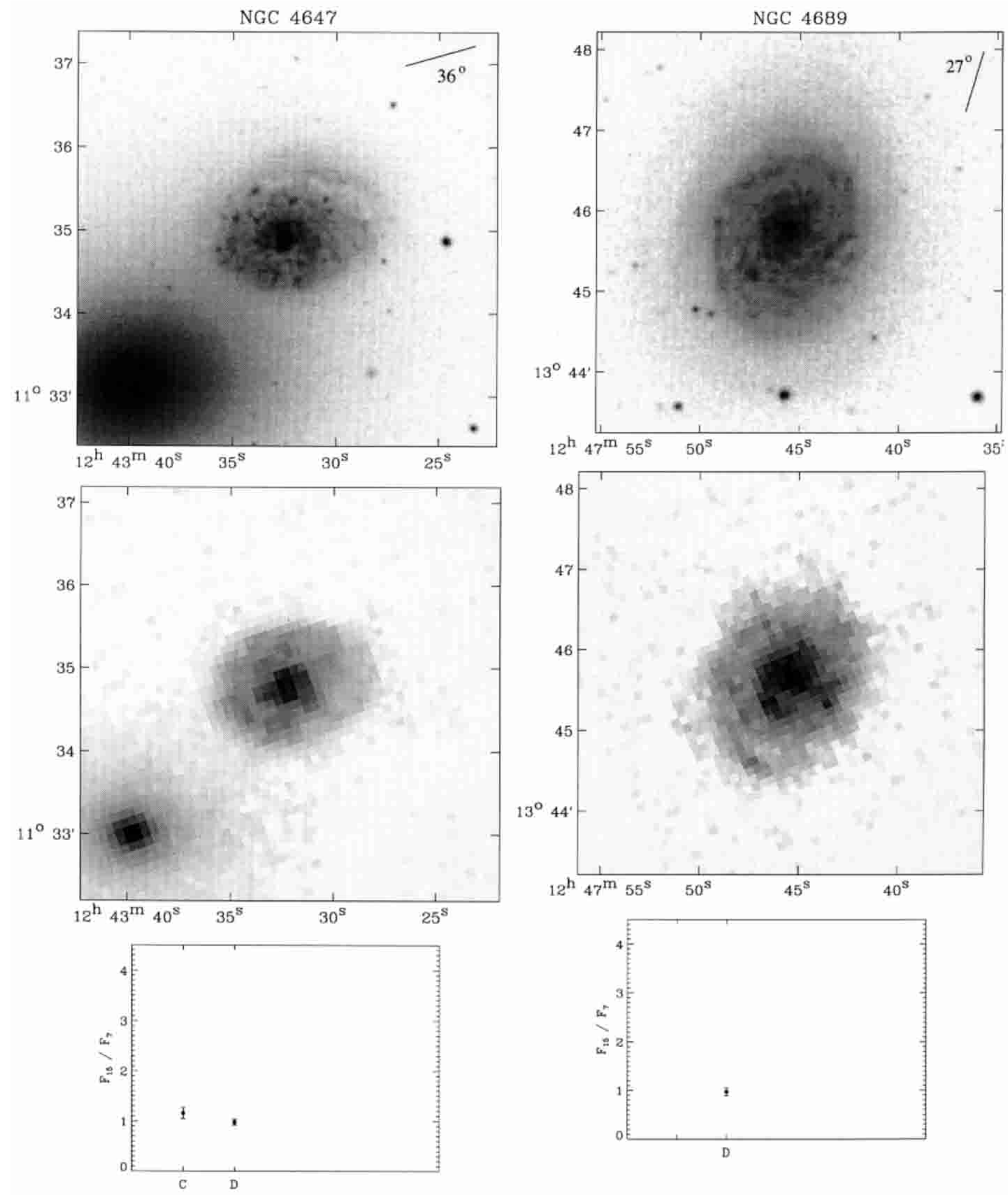

Fig. 9. g) NGC 4647 and 4689 
Acknowledgements. The DSS images shown here were retrieved from the ESO/ST-ECF Archive and were produced at the STScI. They are based on photographic data obtained using the Oschin Schmidt Telescope operated by Caltech and the Palomar Observatory, and the UK Schmidt Telescope operated by the Edinburgh Royal Observatory. The ISOCAM data presented in this paper were analyzed using and adapting the CIA package, a joint development by the ESA Astrophysics Division and the ISOCAM Consortium (led by the PI C. Cesarsky, Direction des Sciences de la Matière, C.E.A., France).

\section{References}

Athanassoula, E. 1992, MNRAS, 259, 345

Athanassoula, E., \& Martinet, L. 1980, A\&A, 87, L10

Ball, R., Sargent, A. I., Scoville, N. Z., Lo, K. Y., \& Scott, S. L. 1985, ApJ, 298, L21

Bonnarel, F., Boulesteix, J., Georgelin, Y. P., et al. 1988, A\&A, 189, 59

Boselli, A., Lequeux, J., Sauvage, M., et al. 1998, A\&A, 335, 53

Boselli, A., Tuffs, R. J., Gavazzi, G., Hippelein, H., \& Pierini, D. 1997, A\&AS, 121, 507

Bosma, A., van der Hulst, J. M., \& Sullivan, W. T. 1977, A\&A, 57,373

Buta, R., \& Crocker, D. A. 1993, AJ, 105, 1344

Buta, R. 1986, ApJS, 61, 631

Cesarsky, C. J., Abergel, A., Agnese, P., et al. 1996, A\&A, 315, L32

Combes, F., Dupraz, C., Casoli, F., \& Pagani, L. 1988, A\&A, 203, L9

Coulais, A., \& Abergel, A. 2000, A\&AS, 141, 533

Dale, D. A., Silbermann, N. A., Helou, G., et al. 2000, AJ, 120, 583

Désert, F.-X., Boulanger, F., \& Puget, J. L. 1990, A\&A, 237, 215

Dottori, H. A., \& Pastoriza, M. G. 1986, RMxAA, 12, 119

Feinstein, C., Vega, I., Mendez, M., \& Forte, J. C. 1990, A\&A, 239, 90

Forbes, D. A., Norris, R. P., Williger, G. M., \& Smith, R. C. 1994, AJ, 107, 984

Förster-Scheiber, N. M., Laurent, O., Sauvage, M., et al. 2001, submitted to A\&A

Friedli, D., Wozniak, H., Rieke, M., Martinet, L., \& Bratschi, P. 1996, A\&AS, 118, 461

García-Barreto, J. A., Franco, J., Carrillo, R., Venegas, S., \& Escalante-Ramírez, B. 1996, RMxAA, 32, 89

García-Barreto, J. A., Franco, J., Guichard, J., \& Carrillo, R. 1995, ApJ, 451, 156

García-Barreto, J. A., Downes, D., Combes, F., et al. 1991, A\&A, 252, 19
Guiderdoni, B., \& Rocca-Volmerange, B. 1985, A\&A, 151, 108

Hameed, S., \& Devereux, N. 1999, AJ, 118, 730

Högbom, J. A. 1974, A\&AS, 15, 417

Jungwiert, B., Combes, F., \& Axon, D. J. 1997, A\&AS, 125, 479

Kenney, J. D. P., \& Lord, S. D. 1991, ApJ, 381, 118

Malhotra, S., Helou, G., van Buren, D., et al. 1996, A\&A, 315, L161

Martin, P., \& Friedli, D. 1997, A\&A, 326, 449

Martin, P., \& Belley, J. 1997, A\&A, 321, 363

Martin, P. 1995, AJ, 109, 2428

Miville-Deschênes, M. A., Boulanger, F., Abergel, A., \& Bernard, J. P. 2000, in ISO beyond point sources: Studies of extended infrared emission, ESA-SP 455, 27

Möllenhoff, C., Matthias, M., \& Gerhard, O. E. 1995, A\&A, 301,359

Morgan, W. W. 1958, PASP, 70, 364

Odewahn, S. C. 1994, AJ, 107, 1320

Pence, W. D., Taylor, K., Freeman, K. C., de Vaucouleurs, G., \& Atherton, P. 1988, ApJ, 326, 564

Prendergast, K. H. 1983, in Internal kinematics and dynamics of galaxies, IAU Symposium, 215

Regan, M. W., Teuben, P. J., \& Vogel, S. N. 1996, AJ, 112, 2549

Regan, M. W., \& Vogel, S. N. 1995, ApJ, 452, L21

Reynaud, D., \& Downes, D. 1998, A\&A, 337, 671

Rice, W., Lonsdale, C. J., Soifer, B. T., et al. 1988, ApJS, 68, 91

Roussel, H., Vigroux, L., Sauvage, M., et al. 2001a, submitted to A\&A, Paper I

Roussel, H., Sauvage, M., \& Vigroux, L. 2001b, submitted to A\&A, Paper II

Sandage, A., \& Bedke, J. 1994, The Carnegie atlas of galaxies

Sandage, A. 1961, The Hubble atlas of galaxies

Sauvage, M., Blommaert, J., Boulanger, F., et al. 1996, A\&A, 315, L89

Sérsic, J. L. 1973, PASP, 85, 103

Sérsic, J. L., \& Pastoriza, M. 1965, PASP, 77, 287

Starck, J. L., Abergel, A., Aussel, H., et al. 1999, A\&AS, 134, 135

Storchi-Bergmann, T., Wilson, A. S., \& Baldwin, J. A. 1996, ApJ, 460, 252

Tagger, M., \& Athanassoula, E. 1991, IAU Symp., 146, 105

Tubbs, A. D. 1982, ApJ, 255, 458

de Vaucouleurs, G., de Vaucouleurs, A., Corwin, H. G., et al. 1991, Third Reference Cat. of Bright Galaxies (RC3)

Vogler, A., Madden, S., Sauvage, M., et al. 2001, in preparation

Wiklind, T., Henkel, C., \& Sage, L. J. 1993, A\&A, 271, 71

Zaritsky, D., \& Lo, K. Y. 1986, ApJ, 303, 66 